\title{
On the classification of Togliatti systems
}

\section{Rosa M. Miró-Roig \& Martí Salat}

To cite this article: Rosa M. Miró-Roig \& Martí Salat (2018) On the classification of Togliatti systems, Communications in Algebra, 46:6, 2459-2475, DOI: 10.1080/00927872.2017.1388813

To link to this article: https://doi.org/10.1080/00927872.2017.1388813

\section{Published online: 08 Nov 2017.}

Submit your article to this journal ๔

\section{山 Article views: 34}

Q View related articles $\asymp$

View Crossmark data $\nearrow$ 


\title{
On the classification of Togliatti systems
}

\author{
Rosa M. Miró-Roig and Martí Salat \\ Department de Matemàtiques i Informàtica, Universitat de Barcelona, Barcelona, Spain
}

\begin{abstract}
In [4], Mezzetti and Miró-Roig proved that the minimal number of generators $\mu(I)$ of a minimal (smooth) monomial Togliatti system $I \subset k\left[x_{0}, \ldots, x_{n}\right]$ satisfies $2 n+1 \leq \mu(I) \leq\left(\begin{array}{c}n+d-1 \\ n-1\end{array}\right)$ and they classify all smooth minimal monomial Togliatti systems $I \subset k\left[x_{0}, \ldots, x_{n}\right]$ with $2 n+1 \leq \mu(I) \leq 2 n+2$. In this paper, we address the first open case. We classify all smooth monomial Togliatti systems $I \subset k\left[x_{0}, \ldots, x_{n}\right]$ of forms of degree $d \geq 4$ with $\mu(I)=2 n+3$ and $n \geq 2$ and all monomial Togliatti systems $I \subset k\left[x_{0}, x_{1}, x_{2}\right]$ of forms of degree $d \geq 6$ with $\mu(I)=7$.
\end{abstract}

\section{ARTICLE HISTORY}

Received 7 March 2017

Revised 21 September 2017

Communicated by R. Piene

\section{KEYWORDS}

Laplace equations; monomial ideals; osculating space; toric varieties; weak Lefschetz property

\section{MATHEMATICS}

SUBJECT CLASSIFICATION 13E10, 14M25, 14N05, $14 \mathrm{~N} 15,53 \mathrm{~A} 20$

\section{Introduction}

The study and classification of smooth projective varieties satisfying at least one Laplace equation is a long standing problem in algebraic geometry. In [6], shedding new light on this subject, it was related to another long standing problem in commutative algebra: the study and classification of homogeneous artinian ideals failing the weak Lefschetz property (WLP). We contribute to these two problems resolving the first question that was left open in [4].

To be more precise. Let $k$ be an algebraically closed field of characteristic $0, R=k\left[x_{0}, \ldots, x_{n}\right]$ and $I=\left(F_{1}, \ldots, F_{s}\right) \subset R$ a homogeneous artinian ideal generated by forms of the same degree $d$. Set $A=R / I$. We say that $A$ fails the WLP from degree $d-1$ to degree $d$ if the homomorphism $\times \ell:[A]_{d-1} \rightarrow[A]_{d}$ induced by a general linear form $\ell$ has not maximal rank. As shown in [6], if $s \leq\left(\begin{array}{c}n+d-1 \\ d\end{array}\right)$ then this assertion is equivalent to saying that the projection $X_{n, d}^{I}$ of the $d$ th Veronese variety $V(n, d) \subset \mathbb{P}^{n}$ from $\left\langle F_{1}, \ldots, F_{s}\right\rangle$ satisfies at least one Laplace equation of order $d-1$. We call Togliatti systems the ideals satisfying these two equivalent statements (see Definition 2.3). The name is in honour of Togliatti who gave a complete classification of rational surfaces parameterized by cubics and satisfying at least one Laplace equation of order 2 (see for instance $[12,13]$ ). Narrowing the field of study we deal only with monomial ideals $I$, so $X_{n, d}^{I}$ turns out to be a toric variety. In this sense, one can apply pure combinatoric tools due to Perkinson in [10] to see whether $I$ is a minimal monomial (smooth) Togliatti system (see Definition 2.3 and Propositions 3.4 and 3.6). In [6], using these tools, Mezzetti, Miró-Roig and Ottaviani classified all smooth minimal monomial Togliatti systems of cubics in four variables and conjectured a further classification for $n \geq 3$. By means of graph theory, this conjecture was proved by Miró-Roig and Michałek [7] where a classification of smooth minimal Togliatti systems $I \subset k\left[x_{0}, \ldots, x_{n}\right]$ of quadrics and cubics is achieved. When $d \geq 4$ the picture becomes much more involved and a complete classification seems out of reach for now. Therefore, in [4] it was introduced another strategy: First to establish lower and upper bounds, depending on $n$ and $d \geq 2$, for the minimal number of generators of a monomial Togliatti system and then to study the monomial Togliatti systems with fixed number of generators. 
In fact, in [4], Mezzetti and Miró-Roig bounded the number of generators of monomial Togliatti systems and classified all minimal monomial (smooth) Togliatti systems reaching the lower bound or close to reach it; namely those generated by $2 n+1$ and $2 n+2$ forms of degree $d \geq 4$. In this paper, we use again combinatoric tools and we classify the first open case, i.e., all minimal monomial Togliatti systems generated by $2 n+3$ forms of degree $d \geq 4$ and $n \geq 2$.

Next we outline the structure of this note. In Section 2 we fix the notation and we collect the basic results needed in the sequel. Then, in Section 3 we expose the main results of this note. First, we give a complete classification of all minimal monomial Togliatti systems generated by 7 forms of degree $d \geq$ 10 in three variables (see Theorem 3.8). In order to achieve this classification we have had to consider all possible configurations of these 7 monomials regarded in the integer standard simplex $d \Delta_{2} \subset \mathbb{Z}^{3}$ and then apply combinatorial criteria to each configuration. Separating the problem in two basic cases which we have also had to separate into a few more subcases has helped so as to reduce the number of configurations to study. Once seen this classification we compute all minimal monomial Togliatti systems generated by 7 forms of degree $6 \leq d \leq 9$ getting a complete scene of what occurs in three variables. From this result we can look apart all minimal monomial smooth Togliatti systems in three variables generated by 7 forms of degree $d \geq 6$ and close the open question we were dealing with.

\section{Preliminaries}

We fix $k$ an algebraically closed field of characteristic zero, $R=k\left[x_{0}, \ldots, x_{n}\right]$ and $\mathbb{P}^{n}=\operatorname{Proj}\left(k\left[x_{0}, \ldots, x_{n}\right]\right)$. Given a homogeneous artinian ideal $I \subset k\left[x_{0}, \ldots, x_{n}\right]$, we denote by $I^{-1}$ the ideal generated by the Macaulay inverse system of $I$ (see [6, Section 3] for details). In this section we fix the notations and the main results that we use throughout this paper. In particular, we quickly recall the relationship between the existence of homogeneous artinian ideals $I \subset k\left[x_{0}, \ldots, x_{n}\right]$ failing the WLP; and the existence of (smooth) projective varieties $X \subset \mathbb{P}^{N}$ satisfying at least one Laplace equation of order $s \geq 2$. For more details, see [6] and [7].

Definition 2.1. Let $I \subset R$ be a homogeneous artinian ideal. We say that $R / I$ has the WLP if there is a linear form $L \in(R / I)_{1}$ such that, for all integers $j$, the multiplication map

$$
\times L:(R / I)_{j} \rightarrow(R / I)_{j+1}
$$

has maximal rank, i.e., it is injective or surjective. We often abuse notation and say that the ideal $I$ has the WLP. If for the general form $L \in(R / I)_{1}$ and for an integer number $j$ the map $\times L$ has not maximal rank we say that the ideal $I$ fails the WLP in degree $j$.

Though many algebras are expected to have the WLP, establishing this property is often rather difficult. For example, it was shown by Stanley [11] and Watanabe [14] that a monomial artinian complete intersection ideal $I \subset R$ has the WLP. By semicontinuity, it follows that a general artinian complete intersection ideal $I \subset R$ has the WLP but it is open whether every artinian complete intersection of height $\geq 4$ over a field of characteristic zero has the WLP. It is worthwhile to point out that the WLP of an artinian ideal $I$ strongly depends on the characteristic of the ground field $k$ and, in positive characteristic, there are examples of artinian complete intersection ideals $I \subset k\left[x_{0}, x_{1}, x_{2}\right]$ failing the WLP (see, e.g. [9, Remark 7.10]).

In [6], Mezzetti et al. showed that the failure of the WLP can be used to construct (smooth) varieties satisfying at least one Laplace equation of order $s \geq 2$ (see also [4, 5, 7]). We have:

Theorem 2.2. Let $I \subset R$ be an artinian ideal generated by $r$ forms $F_{1}, \ldots, F_{r}$ of degree d with $r \leq\left(\begin{array}{c}n+d-1 \\ n-1\end{array}\right)$. The following conditions are equivalent:

(1) the ideal I fails the WLP in degree $d-1$;

(2) the homogeneous forms $F_{1}, \ldots, F_{r}$ become $k$-linearly dependent on a general hyperplane $H$ of $\mathbb{P}^{n}$; 
(3) the closure $X:=\overline{\operatorname{Im}\left(\varphi_{\left.\left(I^{-1}\right)_{d}\right)}\right.} \subset \mathbb{P}^{\left(\begin{array}{c}n+d \\ d\end{array}\right)-r-1}$ of the image of the rational map

$$
\varphi_{\left(I^{-1}\right)_{d}}: \mathbb{P}^{n} \rightarrow \mathbb{P}^{\left(\begin{array}{c}
n+d \\
d
\end{array}\right)-r-1}
$$

associated to $\left(I^{-1}\right)_{d}$ satisfies at least one Laplace equation of order $d-1$.

Proof. See [6, Theorem 3.2].

The above result motivates the following definition:

Definition 2.3. Let $I \subset R$ be an artinian ideal generated by $r$ forms $F_{1}, \ldots, F_{r}$ of degree $d, r \leq\left(\begin{array}{c}n+d-1 \\ n-1\end{array}\right)$. We say:

(i) $I$ is a Togliatti system if it satisfies the three equivalent conditions in Theorem 2.2.

(ii) $I$ is a monomial Togliatti system if, in addition, $I$ (and hence $I^{-1}$ ) can be generated by monomials.

(iii) $I$ is a smooth Togliatti system if, in addition, the $n$-dimensional variety $X$ is smooth.

(iv) A monomial Togliatti system $I$ is minimal if $I$ is generated by monomials $m_{1}, \ldots, m_{r}$ and there is no proper subset $m_{i_{1}}, \ldots, m_{i_{r-1}}$ defining a monomial Togliatti system.

The names are in honor of Eugenio Togliatti who proved that for $n=2$ the only smooth Togliatti system of cubics is $I=\left(x_{0}^{3}, x_{1}^{3}, x_{2}^{3}, x_{0} x_{1} x_{2}\right) \subset k\left[x_{0}, x_{1}, x_{2}\right]$ (see $\left.[12,13]\right)$. This result has been reproved recently by Brenner and Kaid [1] in the context of WLP. Indeed, Togliatti gave a classification of rational surfaces parameterized by cubics and satisfying at least one Laplace equation of order 2: There is only one rational surface in $\mathbb{P}^{5}$ parameterized by cubics and satisfying a Laplace equation of order 2; it is obtained from the 3 rd Veronese embedding $V(2,3)$ of $\mathbb{P}^{2}$ by a suitable projection from four points on it. In [6], the first author together with Mezzetti and Ottaviani classified all smooth rational 3-folds parameterized by cubics and satisfying a Laplace equation of order 2, and gave a conjecture to extend this result to varieties of higher dimension. This conjecture has been recently proved in [7]. Indeed, the first author together with Michałek classified all smooth minimal Togliatti systems of quadrics and cubics. For $d \geq 4$, the picture becomes soon much more involved than in the case of quadrics and cubics, and for the moment a complete classification appears out of reach unless we introduce other invariants as, for example, the number of generators of $I$.

\section{The classification of Togliatti systems with $2 n+3$ generators}

From now on, we restrict our attention to monomial artinian ideals $I \subset k\left[x_{0}, \ldots, x_{n}\right], n \geq 2$, generated by forms of degree $d \geq 4$. It is worthwhile to recall that for monomial artinian ideals to test the WLP there is no need to consider a general linear form. In fact, we have

Proposition 3.1. Let $I \subset R:=k\left[x_{0}, \ldots, x_{n}\right]$ be an artinian monomial ideal. Then $R / I$ has the WLP if and only if $x_{0}+x_{1}+\cdots+x_{n}$ is a Lefschetz element for $R / I$.

Proof. See [9, Proposition 2.2].

Let $I \subset k\left[x_{0}, \ldots, x_{n}\right]$ be a minimal monomial Togliatti systems of forms of degree $d$ and denote by $\mu(I)$ the minimal number of generators of $I$. In [8], the first author and Mezzetti proved:

$$
2 n+1 \leq \mu(I) \leq\left(\begin{array}{c}
n+d-1 \\
n-1
\end{array}\right) .
$$

In addition, the Togliatti systems with number of generators reaching the lower bound or close to the lower bound were classified. Indeed, we have 
Theorem 3.2. Let $I \subset k\left[x_{0}, \ldots, x_{n}\right]$ be a minimal monomial Togliatti system of forms of degree $d \geq 4$. Assume that $\mu(I)=2 n+1$. Then, up to a permutation of the coordinates, one of the following cases holds:

(i) $n \geq 2$ and $I=\left(x_{1}^{d}, \ldots, x_{n}^{d}\right)+x_{0}^{d-1}\left(x_{0}, \ldots, x_{n}\right)$, or

(ii) $\quad(n, d)=(2,5)$ and $I=\left(x_{0}^{5}, x_{1}^{5}, x_{2}^{5}, x_{0}^{3} x_{1} x_{2}, x_{0} x_{1}^{2} x_{2}^{2}\right)$, or

(iii) $\quad(n, d)=(2,4)$ and $I=\left(x_{0}^{4}, x_{1}^{4}, x_{2}^{4}, x_{0} x_{1} x_{2}^{2}, x_{0}^{2} x_{1}^{2}\right)$.

Furthermore, (i) and (ii) are smooth while (iii) is not smooth.

Proof. See [4, Theorem 3.7].

Theorem 3.3. Let $I \subset k\left[x_{0}, \ldots, x_{n}\right]$ be a smooth minimal monomial Togliatti system of forms of degree $d \geq 4$. Assume that $\mu(I)=2 n+2$. Then, up to a permutation of the coordinates, one of the following cases holds:

(i) $n \geq 2$ and $I=\left(x_{0}^{d}, \ldots, x_{n}^{d}\right)+m\left(x_{0}, \ldots, x_{n}\right)$ with $m=x_{0}^{i_{0}} x_{1}^{i_{1}} \cdots x_{n}^{i_{n}}$ where $i_{0} \geq i_{1} \geq \cdots \geq i_{n} \geq 0$, $i_{2}>0$ and $i_{0}+i_{1}+\cdots+i_{n}=d-1$.

(ii) $(n, d)=(2,5)$ and $I=\left(x_{0}^{5}, x_{1}^{5}, x_{2}^{5}, x_{0}^{3} x_{1} x_{2}, x_{0}^{2} x_{1}^{2} x_{2}, x_{0} x_{1}^{3} x_{2}\right)$ or $\left(x_{0}^{5}, x_{1}^{5}, x_{2}^{5}, x_{0}^{3} x_{1} x_{2}, x_{0} x_{1}^{3} x_{2}, x_{0} x_{1} x_{2}^{3}\right)$ or $\left(x_{0}^{5}, x_{1}^{5}, x_{2}^{5}, x_{0}^{2} x_{1}^{2} x_{2}, x_{0}^{2} x_{1} x_{2}^{2}, x_{0} x_{1}^{2} x_{2}^{2}\right)$.

(iii) $(n, d)=(2,7)$ and $d=7$ and $I=\left(x_{0}^{7}, x_{1}^{7}, x_{2}^{7}, x_{0}^{3} x_{1}^{3} x_{2}, x_{0}^{3} x_{1} x_{2}^{3}, x_{0} x_{1}^{3} x_{2}^{3}\right)$ or $\left(x_{0}^{7}, x_{1}^{7}, x_{2}^{7}\right.$, $\left.x_{0}^{5} x_{1} x_{2}, x_{0} x_{1}^{5} x_{2}, x_{0} x_{1} x_{2}^{5}\right),\left(x_{0}^{7}, x_{1}^{7}, x_{2}^{7}, x_{0} x_{1} x_{2}^{5}, x_{0}^{3} x_{1}^{3} x_{2}, x_{0}^{2} x_{1}^{2} x_{2}^{3}\right)$ or $\left(x_{0}^{7}, x_{1}^{7}, x_{2}^{7}, x_{0}^{4} x_{1} x_{2}^{2}, x_{0}^{2} x_{1}^{4} x_{2}, x_{0} x_{1}^{2} x_{2}^{4}\right)$.

Proof. See [4, Theorem 3.17 and Proposition 3.19].

In this paper, we address the first open case and we classify all smooth minimal monomial Togliatti systems $I \subset k\left[x_{0}, \ldots, x_{n}\right]$ of forms of degree $d \geq 4$ with $\mu(I)=2 n+3$ (see Theorem 3.9) as well as all minimal monomial Togliatti systems $I \subset k\left[x_{0}, x_{1}, x_{2}\right]$ of forms of degree $d \geq 6$ with $\mu(I)=7$ (see Theorem 3.8).

In order to achieve this classification, we associate to any artinian monomial ideal a polytope and we tackle our problem with tools coming from combinatorics. In fact, the failure of the WLP of an artinian monomial ideal $I \subset k\left[x_{0}, \ldots, x_{n}\right]$ can be established by purely combinatoric properties of the associated polytope $P_{I}$. To state this result we need to fix some extra notation.

Given an artinian monomial ideal $I \subset k\left[x_{0}, \ldots, x_{n}\right]$ generated by monomials of degree $d$ and its inverse system $I^{-1}$, we denote by $\Delta_{n}$ the standard $n$-dimensional simplex in the lattice $\mathbb{Z}^{n+1}$, we consider $d \Delta_{n}$ and we define the polytope $P_{I}$ as the convex hull of the finite subset $A_{I} \subset \mathbb{Z}^{n+1}$ corresponding to monomials of degree $d$ in $I^{-1}$. As usual we define:

$$
\operatorname{Aff}_{\mathbb{Z}}\left(A_{I}\right):=\left\{\sum_{x \in A_{I}} n_{x} \cdot x \mid n_{x} \in \mathbb{Z}, \quad \sum_{x \in A_{I}} n_{x}=1\right\}
$$

the sublattice $\operatorname{Aff}_{\mathbb{Z}}\left(A_{I}\right)$ in $\mathbb{Z}^{n+1}$ generated by $A_{I}$. We have the following criterion which will play an important role in the proof of our main result.

Proposition 3.4. Let $I \subset k\left[x_{0}, \ldots, x_{n}\right]$ be an artinian monomial ideal generated by monomials of degree $d$. Assume $r \leq\left(\begin{array}{c}n+d-1 \\ n-1\end{array}\right)$. Then, I is a Togliatti system if and only if there exists a hypersurface of degree $d-1$ containing $A_{I} \subset \mathbb{Z}^{n+1}$. In addition, $I$ is a minimal Togliatti system if and only if any such hypersurface $F$ does not contain any integral point of $d \Delta_{n} \backslash A_{I}$ except possibly some of the vertices of $d \Delta_{n}$.

Proof. This follows from Theorem 2.2 and [10, Proposition 1.1].

Example 3.5. The artinian monomial ideal

$$
I=\left(x_{0}, x_{1}\right)^{3}+\left(x_{2}, x_{3}\right)^{3}+\left(x_{4}^{3}, x_{0} x_{2} x_{4}, x_{0} x_{3} x_{4}, x_{1} x_{2} x_{4}, x_{1} x_{3} x_{4}\right) \subset k\left[x_{0}, x_{1}, x_{2}, x_{3}, x_{4}\right]
$$


defines a minimal monomial Togliatti system of cubics. In fact, the set $A_{I} \subset \mathbb{Z}^{5}$ is:

$$
\begin{aligned}
A_{I}= & \{(2,0,1,0,0),(1,0,2,0,0),(2,0,0,1,0),(1,0,0,2,0),(2,0,0,0,1),(1,0,0,0,2),(0,2,1,0,0), \\
& (0,1,2,0,0),(0,2,0,1,0),(0,1,0,2,0),(0,2,0,0,1),(0,1,0,0,2),(0,0,2,0,1),(0,0,1,0,2), \\
& (0,0,0,2,1),(0,0,0,1,2),(1,1,1,0,0),(1,1,0,1,0),(1,1,0,0,1),(1,0,1,1,0),(0,1,1,1,0), \\
& (0,0,1,1,1)\} .
\end{aligned}
$$

There is a quadric, and only one, containing all points of $A_{I}$ and no integral point of $4 \Delta_{4} \backslash A_{I}$, namely,

$$
Q\left(x_{0}, x_{1}, x_{2}, x_{3}, x_{4}\right)=2 \sum_{i=0}^{4} x_{i}^{2}+9\left(x_{0} x_{1}+x_{2} x_{3}\right)-5 \sum_{0 \leq i<j \leq 4} x_{i} x_{j} .
$$

The following criterion allows us to check if a subset $A$ of points in the lattice $\mathbb{Z}^{n+1}$ defines a smooth toric variety $X_{A}$ or not.

Proposition 3.6. Let $I \subset k\left[x_{0}, \ldots, x_{n}\right]$ be an artinian monomial ideal generated by $r$ monomials of degree d. Let $A_{I} \subset \mathbb{Z}^{n+1}$ be the set of integral points corresponding to monomials in $\left(I^{-1}\right)_{d}, S_{I}$ the semigroup generated by $A_{I}$ and $0, P_{I}$ the convex hull of $A_{I}$ and $X_{A_{I}}$ the projective toric variety associated to the polytope $P_{I}$. Then $X_{A_{I}}$ is smooth if and only if for any non-empty face $\Gamma$ of $P_{I}$ the following conditions hold:

(i) The semigroup $S_{I} / \Gamma$ is isomorphic to $\mathbb{Z}_{+}^{m}$ with $m=\operatorname{dim}\left(P_{I}\right)-\operatorname{dim} \Gamma+1$.

(ii) The lattices $\mathbb{Z}^{n+1} \cap A f_{\mathbb{R}}(\Gamma)$ and $A f_{\mathbb{Z}}\left(A_{I} \cap \Gamma\right)$ coincide.

Proof. See [2, Chapter 5, Corollary 3.2]. Note that in this case $X_{A_{I}}=X$ where $X$ is the closure of the image of the rational map $\varphi_{\left[I^{-1}\right]_{d}}: \mathbb{P}^{n} \longrightarrow \mathbb{P}^{\left(\begin{array}{c}n+d \\ d\end{array}\right)-r-1}$.

As a direct application of this criterion we get:

Example 3.7. Let

$$
I=\left(x_{0}, x_{1}\right)^{3}+\left(x_{2}, x_{3}\right)^{3}+\left(x_{4}^{3}, x_{0} x_{2} x_{4}, x_{0} x_{3} x_{4}, x_{1} x_{2} x_{4}, x_{1} x_{3} x_{4}\right) \subset k\left[x_{0}, x_{1}, x_{2}, x_{3}, x_{4}\right]
$$

be the minimal monomial Togliatti system of cubics described in Example 3.5. Applying the above smoothness criterion we get that the toric variety $X_{A_{I}}$ is smooth.

For any integer $d \geq 3$, we define $M(d):=\left\{x_{0}^{a} x_{1}^{b} x_{2}^{c} \mid a+b+c=d\right.$ and $\left.a, b, c \leq d-1\right\}$ and we consider the sets of ideals:

$$
\begin{aligned}
A= & \left\{\left(x_{0}^{2} x_{2}, x_{0} x_{1}^{2}, x_{1}^{3}, x_{1} x_{2}^{2}\right),\left(x_{0}^{2} x_{2}, x_{0} x_{1} x_{2}, x_{1}^{3}, x_{1}^{2} x_{2}\right),\left(x_{0}^{2} x_{2}, x_{0} x_{1} x_{2}, x_{1}^{3}, x_{1} x_{2}^{2}\right),\left(x_{0}^{2} x_{1}, x_{0} x_{2}^{2}, x_{1}^{3}, x_{1}^{2} x_{2}\right),\right. \\
& \left(x_{0}^{2} x_{1}, x_{0} x_{2}^{2}, x_{1}^{3}, x_{1} x_{2}^{2}\right),\left(x_{0}^{2} x_{2}, x_{0} x_{2}^{2}, x_{1}^{3}, x_{1} x_{2}^{2}\right),\left(x_{0} x_{1}^{2}, x_{0} x_{2}^{2}, x_{1}^{3}, x_{1} x_{2}^{2}\right),\left(x_{0}^{2} x_{2}, x_{1}^{2} x_{2}, x_{1}^{3}, x_{2}^{3}\right), \\
& \left(x_{0} x_{2}^{2}, x_{1}^{2} x_{2}, x_{1}^{3}, x_{2}^{3}\right),\left(x_{0}^{2} x_{2}, x_{0} x_{1}^{2}, x_{1}^{2} x_{2}, x_{1} x_{2}^{2}\right),\left(x_{0} x_{1}^{2}, x_{0} x_{2}^{2}, x_{1}^{2} x_{2}, x_{1} x_{2}^{2}\right),\left(x_{0}^{2} x_{1}, x_{0} x_{1}^{2}, x_{1}^{3}, x_{2}^{3}\right), \\
& \left(x_{0}^{2} x_{1}, x_{1}^{2} x_{2}, x_{1}^{3}, x_{2}^{3}\right),\left(x_{1}^{2} x_{2}, x_{1} x_{2}^{2}, x_{1}^{3}, x_{2}^{3}\right),\left(x_{0} x_{2}^{2}, x_{1}^{2} x_{2}, x_{1} x_{2}^{2}, x_{1}^{3}\right), \\
& \left(x_{0} x_{1} x_{2}, x_{0} x_{2}^{2}, x_{1}^{3}, x_{1} x_{2}^{2}\right),\left(x_{0}^{2} x_{2}, x_{0} x_{2}^{2}, x_{1}^{3}, x_{1}^{2} x_{2}\right),\left(x_{0} x_{1}^{2}, x_{0} x_{2}^{2}, x_{1}^{3}, x_{2}^{3}\right), \\
& \left.\left(x_{0}^{2} x_{2}, x_{0} x_{1}^{2}, x_{1}^{3}, x_{2}^{3}\right),\left(x_{0} x_{1} x_{2}, x_{0} x_{2}^{2}, x_{1}^{3}, x_{1}^{2} x_{2}\right),\left(x_{0}^{2} x_{2}, x_{1}^{2} x_{2}, x_{1} x_{2}^{2}, x_{1}^{3}\right)\right\}, \\
B= & \left\{\left(x_{0}^{2} x_{1} x_{2}, x_{0} x_{2}^{3}, x_{1}^{4}, x_{1}^{3} x_{2}\right),\left(x_{0}^{3} x_{2}, x_{0} x_{1}^{2}, x_{2}, x_{1}^{4}, x_{1} x_{2}^{3}\right),\left(x_{0}^{2} x_{2}^{2}, x_{0} x_{1}^{2} x_{2}, x_{1}^{4}, x_{2}^{4}\right),\left(x_{0}^{2} x_{1} x_{2}, x_{1}^{2} x_{2}^{2}, x_{1}^{4}, x_{2}^{4}\right)\right\}
\end{aligned}
$$


and

$$
C=\left\{\left(x_{0}^{2} x_{1} x_{2}^{2}, x_{0} x_{1}^{3} x_{2}, x_{1}^{5}, x_{2}^{5}\right),\left(x_{0}^{3} x_{1} x_{2}, x_{0} x_{1}^{2} x_{2}^{2}, x_{1}^{5}, x_{2}^{5}\right)\right\}
$$

Theorem 3.8. Let $I \subset k\left[x_{0}, x_{1}, x_{2}\right]$ be a minimal monomial Togliatti system of forms of degree $d \geq 10$. Assume that $\mu(I)=7$. Then, up to a permutation of the coordinates, one of the following cases holds:

(1) $I=\left(x_{0}^{d}, x_{1}^{d}, x_{2}^{d}\right)+m\left(x_{0}^{2}, x_{1}^{2}, x_{0} x_{2}, x_{1} x_{2}\right)$ with $m \in M(d-2)$, or

(2) $I=\left(x_{0}^{d}, x_{1}^{d}, x_{2}^{d}\right)+m\left(x_{0}^{2}, x_{1}^{2}, x_{0} x_{1}, x_{2}^{2}\right)$ with $m \in M(d-2)$, or

(3) $I=\left(x_{0}^{d}, x_{1}^{d}, x_{2}^{d}\right)+m\left(x_{0}^{3}, x_{1}^{3}, x_{2}^{3}, x_{0} x_{1} x_{2}\right)$ with $m \in M(d-3)$, or

(4) $I=\left(x_{0}^{d}, x_{1}^{d}, x_{2}^{d}\right)+x_{0}^{d-3} J$ with $J \in A$, or

(5) $I=\left(x_{0}^{d}, x_{1}^{d}, x_{2}^{d}\right)+x_{0}^{d-4} J$ with $J \in B$, or

(6) $I=\left(x_{0}^{d}, x_{1}^{d}, x_{2}^{d}\right)+x_{0}^{d-5} J$ with $J \in C$.

Proof. It is easy to check that all of these ideals are minimal Togliatti systems. Vice versa, let us write $I=\left(x_{0}^{d}, x_{1}^{d}, x_{2}^{d}, m_{1}, m_{2}, m_{3}, m_{4}\right)$ where for $1 \leq i \leq 4, m_{i}=x_{0}^{a_{i}} x_{1}^{b_{i}} x_{2}^{c_{i}}$ with $a_{i}+b_{i}+c_{i}=d$. We consider $A_{I} \subset d \Delta_{2} \cap \mathbb{Z}^{3}$ and we slice $A_{I}$ with planes in three possible manners:

For $0 \leq j \leq 2$ and $0 \leq i \leq d$, we define $H_{i}^{j}:=\left\{\left(t_{0}, t_{1}, t_{2}\right) \mid t_{j}=i\right\}$ and $A_{I}^{(i, j)}:=A_{I} \cap H_{i}^{j}$.

We divide the proof in two cases:

CASE 1: There exist $1 \leq i_{a}, i_{b}, i_{c} \leq 4$ such that $a_{i_{a}}, b_{i_{b}}, c_{i_{c}} \leq 1$.

CASE 2: There exists one variable whose square divides all monomials $m_{i}$.

CASE 1: None of the squares of the variables divide the four monomials $m_{1}, m_{2}, m_{3}$ and $m_{4}$. Up to permutation of the variables, we have two possibilities:

CASE 1A: $I=\left(x_{0}^{d}, x_{1}^{d}, x_{2}^{d}, x_{0}^{e_{1}} x_{1}^{a} x_{2}^{d-a-e_{1}}, x_{0}^{b} x_{1}^{e_{2}} x_{2}^{d-b-e_{2}}, x_{0}^{c} x_{1}^{d-c-e_{3}} x_{2}^{e_{3}}, x_{0}^{\alpha} x_{1}^{\beta} x_{2}^{d-\alpha-\beta}\right)$ with $0 \leq e_{1}, e_{2}, e_{3} \leq$ $1, d-2-e_{1} \geq a \geq 2, d-2-e_{2} \geq b \geq 2, d-2-e_{3} \geq c \geq 2$ and only one of the exponents $\alpha, \beta, d-\alpha-\beta$ is $\leq 1$.

CASE 1B: $I=\left(x_{0}^{d}, x_{1}^{d}, x_{2}^{d}, x_{0}^{e_{1}} x_{1}^{e_{2}} x_{2}^{d-e_{1}-e_{2}}, x_{0}^{a} x_{1}^{d-a-e_{3}} x_{2}^{e_{3}}, x_{0}^{\alpha} x_{1}^{\beta} x_{2}^{d-\alpha-\beta}, x_{0}^{\gamma} x_{1}^{\delta} x_{2}^{d-\gamma-\delta}\right)$ with $0 \leq e_{1}, e_{2}$, $e_{3} \leq 1$.

In both cases, a straightforward computation using the hypothesis $d \geq 10$ shows that when we restrict to $x_{0}+x_{1}+x_{2}$ the 7 monomials remain $k$-linearly independents. Therefore, $I$ is not a Togliatti system.

CASE 2: Without loss of generality we can suppose that $x_{0}^{2}$ divides each monomial $m_{i}$. We can also assume that $a_{1} \geq a_{2} \geq a_{3} \geq a_{4}=s \geq 2$.

Let $F_{d-1}$ be a plane curve of degree $d-1$ containing all integral points of $A_{I}$. Since $s \geq 2$, it factorizes as $F_{d-1}=L_{0}^{0} L_{1}^{0} \cdots L_{s-1}^{0} F_{d-s-1}$. Indeed, $F_{d-1}$ has degree $d-1$ and contains the $d$ points in $A_{I}^{(1,0)}$. So, $F_{d-1}=L_{1}^{0} F_{d-2}$. Since $F_{d-2}$ contains all $d-1$ points of $A_{I}^{(0,0)}$ it factorizes as $F_{d-1}=L_{0}^{0} L_{1}^{0} F_{d-3}$. Repeating the process we arrive to $F_{d-1}=L_{0}^{0} L_{1}^{0} \cdots L_{s-1}^{0} F_{d-s-1}$. We claim that $a_{3}=a_{4}=s \geq 2$. If $a_{3}>a_{4}=s$ then $A_{I}^{(s, 0)}$ has $d-s$ points and $F_{d-s-1}$ contains them. Hence, $F_{d-s-1}=L_{s}^{0} F_{d-s-2}$ contradicting the minimality of $I$ (Proposition 3.4).

So far we have $a_{3}=a_{4}=s \geq 2$ and $F_{d-1}=L_{0}^{0} \cdots L_{s-1}^{0} F_{d-s-1}$ where $F_{d-s-1}$ is a plane curve of degree $d-s-1$ which contains all integer points of $\tilde{A}_{I}:=A_{I} \backslash\left(\cup_{k=0}^{s-1} A_{I}^{(k, 0)}\right)$. Set $\tilde{A}_{I}^{(i, j)}=\tilde{A}_{I} \cap H_{i}^{j}$. We distinguish four subcases: 
CASE 2A: $a_{1}=a_{2}=a_{3}=a_{4}=: s \geq 2$.

CASE 2B: $u:=a_{1}>a_{2}=a_{3}=a_{4}=: s \geq 2$.

CASE 2C: $u:=a_{1}=a_{2}>a_{3}=a_{4}=: s \geq 2$.

CASE 2D: $u:=a_{1}>v:=a_{2}>a_{3}=a_{4}=: s \geq 2$.

CASE 2A: We assume $a_{1}=a_{2}=a_{3}=a_{4}=: s \geq 2$. In this case, $F_{d-1}=L_{0}^{0} \cdots L_{s-1}^{0} L_{s+1}^{0} \cdots L_{d-1}^{0}$. Therefore, $s=d-3$ and $I=\left(x_{0}^{d}, x_{1}^{d}, x_{2}^{d}\right)+x_{0}^{d-3}\left(x_{1}^{3}, x_{1}^{2} x_{2}, x_{1} x_{2}^{2}, x_{2}^{3}\right)$, which is of type (4).

CASE 2B: We assume $u:=a_{1}>a_{2}=a_{3}=a_{4}=: s \geq 2$. In this case, $u \leq s+2$. Indeed, if $u>s+2$ we have, $F_{d-s-1}=L_{s+1}^{0} \cdots L_{u-1}^{0} F_{d-u}$ with $F_{d-u}$ a plane curve of degree $d-u$ which contains in particular $A_{I}^{(s, 0)}$. By minimality, \# $\left(F_{d-u} \cap A_{I}^{(s, 0)}\right)=d-s-2>d-u$ (Proposition 3.4) and we have $F_{d-u}=$ $L_{s}^{0} F_{d-u-1}$, which is a contradiction. Then, up to permutation of variables, $I$ is as one of the following cases:

CASE b1: $u=s+1$ and $I=\left(x_{0}^{d}, x_{1}^{d}, x_{2}^{d}\right)+x_{0}^{s}\left(x_{0} x_{1}^{a} x_{2}^{d-a-s-1}, x_{1}^{b} x_{2}^{d-b-s}, x_{1}^{c} x_{2}^{d-c-s}, x_{1}^{e} x_{2}^{d-e-s}\right)$.

CASE b2: $u=s+2$ and $I=\left(x_{0}^{d}, x_{1}^{d}, x_{2}^{d}\right)+x_{0}^{s}\left(x_{0}^{2} x_{1}^{a} x_{2}^{d-a-s-2}, x_{1}^{b} x_{2}^{d-b-s}, x_{1}^{c} x_{2}^{d-c-s}, x_{1}^{e} x_{2}^{d-e-s}\right)$.

CASE b1: In this case we are removing three points from $H_{s}^{0}$ and one from $H_{s+1}^{0}$. Up to permutation of the variables $y$ and $z$, we can assume $d-s-1 \geq a \geq\left\lfloor\frac{d-s-1}{2}\right\rfloor$ and $d-s \geq b>c>e \geq 0$. Let us first suppose that $\left\lfloor\frac{d-s-1}{2}\right\rfloor>e \geq 0$. In this case

$$
\#\left(F_{d-s-1} \cap \tilde{A}_{I}^{(0,1)}\right)= \begin{cases}d-s & e \geq 1 \\ d-s-1 & e=0 .\end{cases}
$$

If $e \geq 1$, then $\# \tilde{A}_{I}^{(0,1)}=d-s, F_{d-s-1}=L_{0}^{1} F_{d-s-2}=L_{0}^{1} \cdots L_{e-1}^{1} F_{d-s-e-1}$ and $F_{d-s-e-1}$ contains the integer points of $\tilde{A}_{I}^{(e, 1)}$. Since $a>e$ and $b>c>e$, we have \# $\tilde{A}_{I}^{(e, 1)}=d-s-e$ and $F_{d-s-e-1}=$ $L_{e}^{1} F_{d-s-e-2}$ contradicting the minimality of $I$. Therefore, it must be $e=0$, and $m_{4}=x_{0}^{s} x_{2}^{d-s}$ with $d-s-1 \geq c \geq 1$. Let us consider

$$
\#\left(F_{d-s-1} \cap \tilde{A}_{I}^{(1,1)}\right)= \begin{cases}d-s & a, c \geq 2 \\ d-s-1 & a=1, c \geq 2 \\ d-s-1 & a \geq 2, c=1 \\ d-s-2 & a=c=1\end{cases}
$$

and we study the four possibilities.

If $a, c \geq 2$ then we have the factorization $F_{d-s-1}=L_{1}^{1} F_{d-s-2}$. In particular, $F_{d-s-2}$ is a plane curve of degree $d-s-2$ containing the $d-s-1$ points of $\tilde{A}_{I}^{(0,1)}$. So, $F_{d-s-2}=L_{0}^{1} F_{d-s-3}$ which contradicts the minimality of $I$. Therefore, if $a \geq 2$, then $c=1$.

If $a=1$, we have $d-2 \geq s \geq d-3$. If $s=d-2$, then $c=1$ and $I=\left(x_{0}^{d}, x_{1}^{d}, x_{2}^{d}, x_{0}^{d-1} x_{1}\right.$, $\left.x_{0}^{d-2} x_{1}^{2}, x_{0}^{d-2} x_{1} x_{2}, x_{0}^{d-2} x_{2}^{2}\right)$ which is not a Togliatti system. Otherwise, $s=d-3$, then we have several possibilities:

(i) $c \geq 2$ and $I=\left(x_{0}^{d}, x_{1}^{d}, x_{2}^{d}\right)+x_{0}^{d-3}\left(x_{1}^{3}, x_{2}^{3}, x_{0} x_{1} x_{2}\right)+\left(x_{0}^{d-3} x_{1}^{2} x_{2}\right)$ which is not minimal.

(ii) $\quad c=1$ and $I=\left(x_{0}^{d}, x_{1}^{d}, x_{2}^{d}\right)+x_{0}^{d-3}\left(x_{0} x_{1} x_{2}, x_{1}^{3}, x_{2}^{3}\right)+\left(x_{0}^{d-3} x_{1} x_{2}^{2}\right)$ or $I=\left(x_{0}^{d}, x_{1}^{d}, x_{2}^{d}\right)+$ $x_{0}^{d-3} x_{1} x_{2}\left(x_{0}, x_{1}, x_{2}\right)+\left(x_{0}^{d-3} x_{2}^{3}\right)$. Both of them are not minimal. 
If $d-s-1 \geq a \geq 2$ and $s \leq d-3$. We have $e=0, c=1$ and $\left(m_{1}, m_{2}, m_{3}, m_{4}\right)=$ $\left(x_{0}^{s+1} x_{1}^{a} x_{2}^{d-a-s-1}, x_{0}^{s} x_{1}^{b} x_{2}^{d-\bar{b}-s}, x_{0}^{s} x_{1} x_{2}^{d-\bar{s}-1}, x_{0}^{s} x_{2}^{d-s}\right)$ with $d-s \geq b \geq 2$. Let us consider

$$
\#\left(F_{d-s-1} \cap \tilde{A}_{I}^{(0,2)}\right)= \begin{cases}d-s & d-s-1 \geq b, d-s-2 \geq a \\ d-s-1 & a=d-s-1, d-s-1 \geq b \\ d-s-1 & b=d-s, d-s-2 \geq a \\ d-s-2 & b=d-s, a=d-s-1 .\end{cases}
$$

In the first case, we have the factorization $F_{d-s-1}=L_{0}^{2} F_{d-s-2}=L_{0}^{2} L_{1}^{0} F_{d-s-3}$ and it contradicts the minimality of $I$.

If $a=d-s-1$ and $d-s-1 \geq b$, then $b=d-s-1$. Otherwise, we would have $F_{d-s-1}=L_{1}^{2} F_{d-s-2}$ and it would contradict the minimality of $I$. Therefore we have $I=\left(x_{0}^{d}, x_{1}^{d}, x_{2}^{d}\right)+$ $x_{0}^{s}\left(x_{0} x_{1}^{d-s-1}, x_{1}^{d-s-1} x_{2}, x_{1} x_{2}^{d-s-1}, x_{2}^{d-s}\right)$ with $s \leq d-3$. For $s=d-3$ it corresponds to a Togliatti system of type (4), while for $s \leq d-4$ is not Togliatti because when we restrict to $x_{0}+x_{1}+x_{2}=0$ the generators, they remain $k$-linearly independent.

If $d-s-2 \geq a$ and $b=d-s$, then $a=d-s-2$. Hence we have $s \leq d-4$ and $I=\left(x_{0}^{d}, x_{1}^{d}, x_{2}^{d}\right)+$ $x_{0}^{s}\left(x_{0} x_{1}^{d-s-2} x_{2}, x_{1}^{d-s}, x_{1} x_{2}^{d-s-1}, x_{2}^{d-s}\right)$ which is never a Togliatti system.

Finally, if $b=d-s$ and $a=d-s-1$, then $s \leq d-3$ and $I=\left(x_{0}^{d}, x_{1}^{d}, x_{2}^{d}\right)+$ $x_{0}^{s}\left(x_{0} x_{1}^{d-s-1}, x_{1}^{d-s}, x_{1} x_{2}^{d-s-1}, x_{2}^{d-s}\right)$ which is a Togliatti system of type (4) for $s=d-3$ while for $s \leq d-4$ it is not Togliatti.

To finish with the case b1, we have to see what happens when $d-s-2 \geq e \geq\left\lfloor\frac{d-s-1}{2}\right\rfloor$. In this case $s \leq d-3$. Let us see that $a=e$. Otherwise, we can suppose $a>e$ (the other case is symmetric) and we have the factorization $F_{d-s-1}=L_{0}^{1} \cdots L_{e-1}^{1} F_{d-s-e-1}$. Since $a>e$ and $b>c>e, \tilde{A}_{I}^{(e, 1)}$ has $d-s-e$ points and we have the factorization $F_{d-s-e-1}=L_{e}^{1} F_{d-s-e-2}$ which contradicts the minimality of $I$. Hence $a=e$ and in particular $d-s-1>a$ and $d-s \geq b>c>a$.

Let us consider $\tilde{\tilde{A}}_{I}:=\tilde{A}_{I} \backslash\left(\cup_{k=0}^{a-1}\right)$ in the same spirit as $A_{I}$ and $\tilde{A}_{I}$. If $b=d-s$, then $\tilde{\tilde{A}}_{I}^{(0,2)}$ consists in $d-s-e$ different points. Otherwise, $d-s-1 \geq b$ and $\# \tilde{\tilde{A}}_{I}^{(0,2)}=d-s+1-e$. In both cases $\tilde{\tilde{A}}_{I}^{(0,2)}$ have more points than the degree of the curve $F_{d-s-e-1}$ which passes through them. Therefore, $F_{d-s-e-1}=$ $L_{0}^{2} F_{d-s-e-2}$ and $d-s-1 \geq b$. Since $m_{2}$ cannot be aligned vertically with any other monomial $m_{i}$, we can repeat this argument until we get that $b=c+1$ and $F_{d-s-e-1}=L_{0}^{2} \cdots L_{b-1}^{2} F_{d-s-e-b-1}$. Now $F_{d-s-e-b-1}$ is a plane curve of degree $d-s-e-b-1$ containing all $d-s-e-b$ points of $\tilde{\tilde{A}}_{I}^{(b, 2)}$. Hence, we can factorize $F_{d-s-e-b-1}=L_{b}^{2} F_{d-s-e-b-2}$ contradicting the minimality assumption.

CASE b2: We are removing from $d \Delta_{2}$ to get $\tilde{A}_{I}$ : three points of $H_{s}^{0}$ and one from $H_{s+2}^{0}$. Up to permutation of the variables $y$ and $z$, we can suppose that $d-s-2 \geq a \geq\left\lfloor\frac{d-s-2}{2}\right\rfloor$ and $d-s \geq b>c>e \geq 0$.

Let us suppose first that $\left\lfloor\frac{d-s-2}{2}\right\rfloor>e \geq 0$. We argue as in the case $u=s+1$ to prove that $e=0$. Let us consider \# $\left(F_{d-s-1} \cap \tilde{A}_{I}^{(1,1)}\right)$. Using the same argumentation we prove that if $a, c \geq 2$ we get a contradiction. If $a=1$, then either $s=d-3$ or $s=d-4$ and we have the following cases:

(i) If $s=d-3$, then $\left(m_{1}, m_{2}, m_{3}, m_{4}\right)$ is $x_{0}^{d-3}\left(x_{0}^{2} x_{1}, x_{1}^{3}, x_{1}^{2} x_{2}, x_{2}^{3}\right), x_{0}^{d-3}\left(x_{0}^{2} x_{1}, x_{1}^{3}, x_{1} x_{2}^{2}, x_{2}^{3}\right)$ or $x_{0}^{d-3}\left(x_{0}^{2} x_{1}, x_{1}^{2} x_{2}, x_{1} x_{2}^{2}, x_{2}^{3}\right)$. All of them are Togliatti systems of type (4).

(ii) If $s=d-4$, then $\left(m_{1}, m_{2}, m_{3}, m_{4}\right)$ is $x_{0}^{d-4}\left(x_{0}^{2} x_{1} x_{2}, x_{1}^{4}, x_{1}^{3} x_{2}, x_{2}^{4}\right), x_{0}^{d-4}\left(x_{0}^{2} x_{1} x_{2}, x_{1}^{4}, x_{1}^{2} x_{2}^{2}, x_{2}^{4}\right)$, $x_{0}^{d-4}\left(x_{0}^{2} x_{1} x_{2}, x_{1}^{4}, x_{1} x_{2}^{3}, x_{2}^{4}\right), \quad x_{0}^{d-4}\left(x_{0}^{2} x_{1} x_{2}, x_{1}^{3} x_{2}, x_{1}^{2} x_{2}^{2}, x_{2}^{4}\right), \quad x_{0}^{d-4}\left(x_{0}^{2} x_{1} x_{2}, x_{1}^{3} x_{2}, x_{1} x_{2}^{3}, x_{2}^{4}\right) \quad$ or $x_{0}^{d-4}\left(x_{0}^{2} x_{1} x_{2}, x_{1}^{2} x_{2}^{2}, x_{1} x_{2}^{3}, x_{2}^{4}\right)$. The only one which is a minimal Togliatti system is the second one and it is of type (5).

Now, we assume $e=0, c=1$ and $d-s-2 \geq a \geq 2$. In particular, $s \leq d-4$. We consider $\#\left(F_{d-s-1} \cap \tilde{A}_{I}^{(0,2)}\right)$, and see that if $d-s-1 \geq b \geq 2$ and $d-s-3 \geq a \geq 2$, there is a contradiction with the minimality of $I$. If $b=d-s$ and $a \leq d-s-3$ (resp. $a=d-s-2$ and $b \leq d-s-1$ ) 
then $a=d-s-3$ (resp. $b=d-s-1$ ). Otherwise we would incur again to a contradiction with the minimality of $I$. So, we have three possibilities.

(i) $a=d-s-3 \geq 2, b=d-s, s \leq d-5$ and $I=\left(x_{0}^{d}, x_{1}^{d}, x_{2}^{d}\right)+x_{0}^{s}\left(x_{0}^{2} x_{1}^{d-s-3} x_{2}, x_{1}^{d-s}, x_{1} x_{2}^{d-s-1}, x_{2}^{d-s}\right)$.

(ii) $a=d-s-2, b=d-s-1, s \leq d-4$ and $I=\left(x_{0}^{d}, x_{1}^{d}, x_{2}^{d}\right)+x_{0}^{s}\left(x_{0}^{2} x_{1}^{d-s-2}, x_{1}^{d-s-1} x_{2}, x_{1} x_{2}^{d-s-1}, x_{2}^{d-s}\right)$.

(iii) $\quad a=d-s-2, b=d-s, s \leq d-4$ and $I=\left(x_{0}^{d}, x_{1}^{d}, x_{2}^{d}\right)+x_{0}^{s}\left(x_{0}^{2} x_{1}^{d-s-2}, x_{1}^{d-s}, x_{1} x_{2}^{d-s-1}, x_{2}^{d-s}\right)$.

After restricting to $x_{0}+x_{1}+x_{2}=0$, we see that none of them corresponds to a Togliatti system.

To finish with the case b2, we see what happens when $d-s-2 \geq e \geq\left\lfloor\frac{d-s-2}{2}\right\rfloor$. With the same argument that we use before, we can see that $a=e$. The difference with the case $u=s+1$ is that in this case we can have $m_{1}$ and $m_{2}$ aligned vertically. This condition can be translated as the case when $d-b-s=d-a-s-2$. If this does not happen (i.e. $b>a+2$ ), then it will contradict the minimality of $I$. Indeed: let us suppose that $0 \leq k:=d-b-s<d-a-s-2$. Inductively we have the factorization $F_{d-s-e-1}=L_{0}^{2} \cdots L_{k-1}^{2} F_{d-s-e-k-1} \cdot F_{d-s-e-k-1}$ is a plane curve of degree $d-s-e-k-1$ which passes through all $d-s-e-k$ points of $\tilde{\tilde{A}}_{I}^{k}$. Hence, we have the factorization $F_{d-s-e-k-1}=L_{k}^{2} F_{d-s-e-k-2}$, contradicting the minimality assumption.

Therefore it must be $b=a+2$ and, since $b>c>a$ we have $c=a+1$. Finally we get: $I=$ $\left(x_{0}^{d}, x_{1}^{d}, x_{2}^{d}\right)+x_{0}^{s} x_{1}^{a} x_{2}^{d-a-s-2}\left(x_{0}^{2}, x_{1}^{2}, x_{1} x_{2}, x_{2}^{2}\right)$ which is of type (1).

CASE 2C: We assume that $u:=a_{1}=a_{2}>a_{3}=a_{4}=: s \geq 2$. Arguing as in case 2B we get $u=s+1$ and $I=\left(x_{0}^{d}, x_{1}^{d}, x_{2}^{d}\right)+x_{0}^{s}\left(x_{0} x_{1}^{a} x_{2}^{d-a-s-1}, x_{0} x_{1}^{b} x_{2}^{d-b-s-1}, x_{1}^{c} x_{2}^{\bar{d}-c-s}, x_{1}^{e} x_{2}^{d-e-s}\right)$. We can assume $d-s-1 \geq$ $a \geq\left\lfloor\frac{d-s-1}{2}\right\rfloor, a>b$ and $d-s \geq c>e \geq 0$.

Let us suppose first that $\left\lfloor\frac{d-s-1}{2}\right\rfloor>e \geq 0$. We consider

$$
\#\left(F_{d-s-1} \cap \tilde{A}_{I}^{(0,1)}\right)=\left\{\begin{array}{lll}
d-s & e \geq 1, b \geq 1 & c 1 \\
d-s-1 & e=0, b \geq 1 & c 2 \\
d-s-1 & e \geq 1, b=0 & c 3 \\
d-s-2 & e=b=0 & c 4
\end{array}\right.
$$

CASE C1: Since $F_{d-s-1}$ is a plane curve of degree $d-s-1$ which contains all $d-s$ points of $\tilde{A}_{I}^{(0,1)}$ we have the factorization $F_{d-s-1}=L_{0}^{1} F_{d-s-2}$. Now, intersecting $F_{d-s-2}$ with $\tilde{A}_{I}^{(1,1)}$ and using the minimality assumption, we see that the only two possibilities are either $e \geq 2$ and $b \geq 2$ or $e=b=1$. In the first case $F_{d-s-2}$ factorizes as $F_{d-s-2}=L_{1}^{1} F_{d-s-3}$. Repeating the same argument we get that it must be $e=b$ in any case. Now, we consider $\tilde{\tilde{A}}_{I}$ as before and we take

$$
\#\left(F_{d-s-e-1} \cap \tilde{\tilde{A}}_{I}^{(0,2)}\right)= \begin{cases}d-s-e+1 & d-s-2 \geq a, d-s-1 \geq c \\ d-s-e & a=d-s-1, d-s-1 \geq c \\ d-s-e & d-s-2 \geq a, c=d-s \\ d-s-e-1 & a=d-s-1, c=d-s\end{cases}
$$

In the second and third cases we obtain directly a contradiction with the minimality of $I$. In the fourth case, $\tilde{\tilde{A}}_{I}^{(1,2)}$ consists in $d-s-e$ different points and we have $F_{d-s-e-1}=L_{1}^{2} F_{d-s-e-2}$. Since $\tilde{\tilde{A}}_{I}^{(0,2)}$ has $d-s-e-1$ different points, we get a contradiction with the minimality of I. Finally, in the first case we obtain a factorization $F_{d-s-e-1}=L_{0}^{2} F_{d-s-e-2}$ and we repeat the same argument until we get that $m_{1}$ and $m_{3}$ are always vertically aligned. Then, $c=a+1$ and we have the factorization $F_{d-s-e-1}=L_{0}^{2} \cdots L_{d-s-a-2}^{2} F_{a-e}$. If $a \geq e+2$ we have the factorization $F_{a-e}=L_{e+1}^{1} \cdots L_{a-1}^{1} F_{1}$, which contradicts the minimality of $I$. Therefore, $a=e+1$ 
and $I=\left(x_{0}^{d}, x_{1}^{d}, x_{2}^{d}\right)+x_{0}^{s}\left(x_{0} x_{1}^{e+1} x_{2}^{d-s-e-2}, x_{0} x_{1}^{e} x_{2}^{d-s-e-1}, x_{1}^{e+2} x_{2}^{d-s-e-2}, x_{1}^{e} x_{2}^{d-s-e}\right)=\left(x_{0}^{d}, x_{1}^{d}, x_{2}^{d}\right)+$ $x_{0}^{s} x_{1}^{e} x_{2}^{d-s-e-2}\left(x_{0} x_{1}, x_{0} x_{2}, x_{1}^{2}, x_{2}^{2}\right)$ which is of type (1).

CASE C2: We assume $e=0$ and $b \geq 1$. Let us consider

$$
\#\left(F_{d-s-1} \cap \tilde{A}_{I}^{(1,1)}\right)= \begin{cases}d-s & b \geq 2, c \geq 2 \\ d-s-1 & b=1, c \geq 2 \\ d-s-1 & b \geq 2, c=1 \\ d-s-2 & b=c=1\end{cases}
$$

In Case (i) we factorize $F_{d-s-1}=L_{1}^{1} F_{d-s-2}$. Since $F_{d-s-2}$ is a plane curve of degree $d-s-2$ containing all $d-s-1$ different points of $\tilde{A}_{I}^{(0,1)}$ it factorizes as $F_{d-s-2}=L_{0}^{1} F_{d-s-3}$. This contradicts the minimality of $I$.

Case (ii): assume $e=0, b=1$ and $c \geq 2$. We consider $\#\left(F_{d-s-1} \cap \tilde{A}_{I}^{(0,2)}\right)$ and arguing as in the previous cases we get three possibilities:

$$
\begin{aligned}
& a=d-s-1, c=d-s-1 \text { and } I=\left(x_{0}^{d}, x_{1}^{d}, x_{2}^{d}\right)+x_{0}^{s}\left(x_{0} x_{1}^{d-s-1}, x_{0} x_{1} x_{2}^{d-s-2}, x_{1}^{d-s-1} x_{2}, x_{2}^{d-s}\right) . \\
& a=d-s-2, c=d-s \text { and } I=\left(x_{0}^{d}, x_{1}^{d}, x_{2}^{d}\right)+x_{0}^{s}\left(x_{0} x_{1}^{d-s-2} x_{2}, x_{0} x_{1} x_{2}^{d-s-2}, x_{1}^{d-s}, x_{2}^{d-s}\right) . \\
& a=d-s-1, c=d-s \text { and } I=\left(x_{0}^{d}, x_{1}^{d}, x_{2}^{d}\right)+x_{0}^{s}\left(x_{0} x_{1}^{d-s-1}, x_{0} x_{1} x_{2}^{d-s-2}, x_{1}^{d-s}, x_{2}^{d-s}\right) .
\end{aligned}
$$

Restricting the generators to the hyperplane $x_{0}+x_{1}+x_{2}$, we see that each of them is a Togliatti system if, and only if $s=d-3$.

Case (iii): assume $e=0, c=1$ and $b \geq 2$. In particular $a \geq 3$ and $s \leq d-4$. Arguing as before, we see that the only viable possibility is $a=d-s-1$ and $b=d-s-2$. Therefore $I=\left(x_{0}^{d}, x_{1}^{d}, x_{2}^{d}\right)+$ $x_{0}^{s}\left(x_{0} x_{1}^{d-s-1}, x_{0} x_{1}^{d-s-3} x_{2}^{2}, x_{1} x_{2}^{d-s-1}, x_{2}^{d-s}\right)$, which is never a Togliatti system.

Case (iv): assume $e=0$ and $b=c=1$. Now it only remains to determinate $a$. If $d-s-2 \geq a$, we have $F_{d-s-1}=L_{0}^{2} \cdots L_{d-s-a-2}^{2} F_{a}$. Since $\tilde{A}_{I}^{(d-s-a-1,2)}$ consists in $a+1$ different points, we get a contradiction with the minimality of $I$. Therefore, $a=d-s-1$. Using the same argumentation we see that $a=b+1=2$. Thus $s=d-4$ and $I=\left(x_{0}^{d}, x_{1}^{d}, x_{2}^{d}\right)+x_{0}^{d-4}\left(x_{0} x_{1}^{3}, x_{0} x_{1} x_{2}^{2}, x_{1} x_{2}^{3}, x_{2}^{4}\right)$ which is not a Togliatti system.

CASE C3: Now, assume that $b=0$ and $e \geq 1$. Since $a>e$ by hypothesis, considering $\#\left(F_{d-s-1} \cap \tilde{A}_{I}^{(1,1)}\right)$ we see that $e=1$ and $s \leq d-3$. If we consider $\#\left(F_{d-s-1} \cap \tilde{A}_{I}^{(0,2)}\right)$ we get that the only viable possibilities are:

$$
\begin{aligned}
& a=d-s-1, c=d-s-1 \text { and } I=\left(x_{0}^{d}, x_{1}^{d}, x_{2}^{d}\right)+x_{0}^{s}\left(x_{0} x_{1}^{d-s-1}, x_{0} x_{2}^{d-s-1}, x_{1}^{d-s-1} x_{2}, x_{1} x_{2}^{d-s-1}\right) \\
& a=d-s-2, c=d-s \text { and } I=\left(x_{0}^{d}, x_{1}^{d}, x_{2}^{d}\right)+x_{0}^{s}\left(x_{0} x_{1}^{d-s-2} x_{2}, x_{0} x_{2}^{d-s-1}, x_{1}^{d-s}, x_{1} x_{2}^{d-s-1}\right) . \\
& a=d-s-1, c=d-s \text { and } I=\left(x_{0}^{d}, x_{1}^{d}, x_{2}^{d}\right)+x_{0}^{s}\left(x_{0} x_{1}^{d-s-1}, x_{0} x_{2}^{d-s-1}, x_{1}^{d-s}, x_{1} x_{2}^{d-s-1}\right) .
\end{aligned}
$$

Each of them are Togliatti systems if, and only if $s=d-3$.

CASE C4: in this case we assume that $e=b=0$. If $a, c \geq 3$, we have the factorization $F_{d-s-1}=$ $L_{1}^{1} L_{2}^{1} F_{d-s-3}$. Since $F_{d-s-3}$ is a plane curve containing all $d-s-2$ points of $\tilde{A}_{I}^{(0,1)}$, we get $F_{d-s-3}=$ $L_{0}^{0} F_{d-s-4}$ which contradicts the minimality of $I$. Therefore we can consider three subcases:

Case (i). Assume that $a=1$, then it has to be either $s=d-2$ or $s=d-3$. Hence $I$ is one of the following possibilities: $\left(x_{0}^{d}, x_{1}^{d}, x_{2}^{d}\right)+x_{0}^{d-2}\left(x_{0} x_{1}, x_{0} x_{2}, x_{1}^{2}, x_{2}^{2}\right),\left(x_{0}^{d}, x_{1}^{d}, x_{2}^{d}\right)+x_{0}^{d-2} x_{2}\left(x_{0}, x_{1}, x_{2}\right)+\left(x_{0}^{d-1} x_{1}\right)$, $\left(x_{0}^{d}, x_{1}^{d}, x_{2}^{d}\right)+x_{0}^{d-3}\left(x_{0} x_{1} x_{2}, x_{0} x_{2}^{2}, x_{1}^{3}, x_{2}^{3}\right),\left(x_{0}^{d}, x_{1}^{d}, x_{2}^{d}\right)+x_{0}^{d-3} x_{2}\left(x_{0} x_{1}, x_{0} x_{2}, x_{1}^{2}, x_{2}^{2}\right)$ and $\left(x_{0}^{d}, x_{1}^{d}, x_{2}^{d}\right)+$ 
$x_{0}^{d-3} x_{2}^{2}\left(x_{0}, x_{1}, x_{2}\right)+\left(x_{0}^{d-2} x_{1} x_{2}\right)$. And only the first and the third possibilities give to minimal Togliatti systems of type (1) and type (4) respectively.

Case (ii). Assume that $a=2$, then it can be either $s=d-3, s=d-4$ or $s=d-$ 5. Therefore, $I$ is one of the next ideals: $\left(x_{0}^{d}, x_{1}^{d}, x_{2}^{d}\right)+x_{0}^{d-3}\left(x_{0} x_{1}^{2}, x_{0} x_{2}^{2}, x_{1}^{3}, x_{2}^{3}\right),\left(x_{0}^{d}, x_{1}^{d}, x_{2}^{d}\right)+$ $x_{0}^{d-3}\left(x_{0} x_{1}^{2}, x_{0} x_{2}^{2}, x_{1}^{2} x_{2}, x_{2}^{3}\right),\left(x_{0}^{d}, x_{1}^{d}, x_{2}^{d}\right)+x_{0}^{d-3}\left(x_{0}, x_{1}, x_{2}\right)+\left(x_{0}^{d-2} x_{1}^{2}\right),\left(x_{0}^{d}, x_{1}^{d}, x_{2}^{d}\right)+x_{0}^{d-4}\left(x_{0} x_{1}^{2} x_{2}\right.$, $\left.x_{0} x_{2}^{3}, x_{1}^{4}, x_{2}^{4}\right),\left(x_{0}^{d}, x_{1}^{d}, x_{2}^{d}\right)+x_{0}^{d-4}\left(x_{0} x_{1}^{2} x_{2}, x_{0} x_{2}^{3}, x_{1}^{3} x_{2}, x_{2}^{4}\right),\left(x_{0}^{d}, x_{1}^{d}, x_{2}^{d}\right)+x_{0}^{d-4}\left(x_{0} x_{1}^{2} x_{2}, x_{0} x_{2}^{3}, x_{1}^{2} x_{2}^{2}, x_{2}^{4}\right)$, $\left(x_{0}^{d}, x_{1}^{d}, x_{2}^{d}\right)+x_{0}^{d-4} x_{2}^{3}\left(x_{0}, x_{1}, x_{2}\right)+\left(x_{0}^{d-3} x_{1}^{2} x_{2}\right),\left(x_{0}^{d}, x_{1}^{d}, x_{2}^{d}\right)+x_{0}^{d-5}\left(x_{0} x_{1}^{2} x_{2}^{2}, x_{0} x_{2}^{4}, x_{1}^{5}, x_{2}^{5}\right),\left(x_{0}^{d}, x_{1}^{d}, x_{2}^{d}\right)+$ $x_{0}^{d-5}\left(x_{0} x_{1}^{2} x_{2}^{2}, x_{0} x_{2}^{4}, x_{1}^{4} x_{2}, x_{2}^{5}\right),\left(x_{0}^{d}, x_{1}^{d}, x_{2}^{d}\right)+x_{0}^{d-5}\left(x_{0} x_{1}^{2} x_{2}^{2}, x_{0} x_{2}^{4}, x_{1}^{3} x_{2}^{2}, x_{2}^{5}\right),\left(x_{0}^{d}, x_{1}^{d}, x_{2}^{d}\right)+x_{0}^{d-5}\left(x_{0} x_{1}^{2} x_{2}^{2}, x_{0} x_{2}^{4}\right.$, $\left.x_{1}^{2} x_{2}^{3}, x_{2}^{5}\right)$ and $\left(x_{0}^{d}, x_{1}^{d}, x_{2}^{d}\right)+x_{0}^{d-5} x_{2}^{4}\left(x_{0}, x_{1}, x_{2}\right)+\left(x_{0}^{d-4} x_{1}^{2} x_{2}^{2}\right)$.

Only the first and the second ones correspond to minimal Togliatii systems.

Case (iii). Assume that $a \geq 3$ which implies that either $c=1$ or $c=2$ and we have $s \leq d-4$. In both cases, since $a \geq 3$ and $c \leq 2, m_{1}$ cannot be aligned vertically with any $m_{i}$. Therefore, in both cases, we get a contradiction with the minimality of $I$.

To finish case $2 \mathrm{C}$, let us assume $e \geq\left\lfloor\frac{d-s-1}{2}\right\rfloor>0$. We will separate two cases: when $b=0$ and when $b \geq 1$.

Case (i). We assume $b=0$, then considering $\#\left(F_{d-s-1} \cap \tilde{A}_{I}^{(1,1)}\right)$ and using the bound for $e$, we obtain that $a=1$ and therefore it is either $s=d-2$ or $d-3$. So, $I$ is one of the following ideals: $\left(x_{0}^{d}, x_{1}^{d}, x_{2}^{d}\right)+x_{0}^{d-2} x_{1}\left(x_{0}, x_{1}, x_{2}\right)+\left(x_{0}^{d-1} x_{2}\right),\left(x_{0}^{d}, x_{1}^{d}, x_{2}^{d}\right)+x_{0}^{d-3}\left(x_{0} x_{1} x_{2}, x_{0} x_{2}^{2}, x_{1}^{3}, x_{1}^{2} x_{2}\right),\left(x_{0}^{d}, x_{1}^{d}, x_{2}^{d}\right)+$ $x_{0}^{d-3}\left(x_{0} x_{1} x_{2}, x_{0} x_{2}^{2}, x_{1}^{3}, x_{1} x_{2}^{2}\right)$ and $\left(x_{0}^{d}, x_{1}^{d}, x_{2}^{d}\right)+x_{0}^{d-3} x_{1} x_{2}\left(x_{0}, x_{1}, x_{2}\right)+\left(x_{0}^{d-2} x_{2}^{2}\right)$. And any of them are minimal Togliatti systems.

Case (ii). We assume $b \geq 1$. In this case, we can assume $e \geq b$ (the other case is symmetric) and we obtain the factorization $F_{d-s-1}=L_{0}^{1} \cdots L_{b-1}^{1} F_{d-s-b-1}$. If $e>b$ we get a contradiction with the minimality of $I$. Hence, $e=b$. Now we consider as before $\tilde{\tilde{A}}_{I}$ and we have

$$
\#\left(F_{d-s-b-1} \cap \tilde{\tilde{A}}_{I}^{(0,2)}\right)= \begin{cases}d-s-b+1 & d-s-2 \geq a, d-s-1 \geq c \\ d-s-b & a=d-s-1, d-s-1 \geq c \\ d-s-b & d-s-2 \geq a, c=d-s \\ d-s-b-1 & a=d-s-1, c=d-s\end{cases}
$$

In the second and third cases we get immediately a contradiction with the minimality. In the first case, we can repeat the same argument and we get contradiction unless $m_{1}$ and $m_{3}$ are aligned vertically. Hence, we always obtain that $c=a+1$, and we have the factorization $F_{d-s-b-1}=L_{0}^{2} \cdots L_{d-s-a-2}^{2} F_{a-b}$. If $a \geq b+2$, then $\tilde{\tilde{A}}_{I}^{(d-s-a, 2)}$ consists in $a-b+1$ different points, so we have the factorization $F_{a-b}=L_{d-s-a}^{2} F_{a-b-1}$. Now $F_{a-b-1}$ is a plane curve of degree $a-b-1$ which contains all $a-b$ points of $\tilde{\tilde{A}}_{I}^{(d-s-a-1,2)}$ and then it factorizes as $F_{a-b-1}=L_{d-s-a-1}^{2} F_{a-b-2}$ which contradicts the minimality of $I$. Therefore, $a=b+1$ and $I=\left(x_{0}^{d}, x_{1}^{d}, x_{2}^{d}\right)+x_{0}^{s}\left(x_{0} x_{1}^{b+1} x_{2}^{d-s-b-2}, x_{0} x_{1}^{b} x_{2}^{d-s-b-1}, x_{1}^{b+2} x_{2}^{d-s-b-2}, x_{1}^{b} x_{2}^{d-s-b}\right)=\left(x_{0}^{d}, x_{1}^{d}, x_{2}^{d}\right)+$ $x_{0}^{s} x_{1}^{b} x_{2}^{d-s-b-2}\left(x_{0} x_{1}, x_{0} x_{2}, x_{1}^{2}, x_{2}^{2}\right)$ which is of type (1).

CASE 2D: We assume that $u:=a_{1}>v:=a_{2}>a_{3}=a_{4}=: s \geq 2$. Recall that we have the factorization $F_{d-1}=L_{0}^{0} L_{1}^{0} \cdots L_{s-1}^{0} F_{d-s-1}$ and we easily check that the minimality of $I$ forces $v=s+1$. So we can write $I=\left(x_{0}^{d}, x_{1}^{d}, x_{2}^{d}\right)+x_{0}^{s}\left(x_{0}^{r} x_{1}^{a} x_{2}^{d-a-s-r}, x_{0} x_{1}^{b} x_{2}^{d-b-s-1}, x_{1}^{c} x_{2}^{d-c-s}, x_{1}^{e} x_{2}^{d-e-s}\right)$ with $u=s+r$ and $d-s-1 \geq r \geq 2$. We can assume $d-s-1 \geq b \geq\left\lfloor\frac{d-s-1}{2}\right\rfloor$ and $d-s \geq c>e \geq 0$, and we have 
$d-s-r \geq a \geq 0$ and $s \leq d-3$. Let us suppose first that $\left\lfloor\frac{d-s-1}{2}\right\rfloor>e \geq 0$. We consider

$$
\#\left(F_{d-s-1} \cap \tilde{A}_{I}^{(0,1)}\right)=\left\{\begin{array}{lll}
d-s & e \geq 1, a \geq 1 & (d 1) \\
d-s-1 & e=0, a \geq 1 & (d 2) \\
d-s-1 & e \geq 1, a=0 & (d 3) \\
d-s-2 & e=a=0
\end{array}\right.
$$

CASE d1: In this case $a=e$. Indeed, if $a>e \geq 1$ (the other case is symmetric) we have the factorization $F_{d-s-1}=L_{0}^{1} \ldots L_{e-1}^{1} F_{d-s-e-1}=L_{0}^{1} \ldots L_{e-1}^{1} L_{e}^{1} F_{d-s-e-2}$ which contradicts the minimality of $I$. Let us now consider

$$
\#\left(F_{d-s-e-1} \cap \tilde{A}_{I}^{(e+1,1)}\right)= \begin{cases}d-s-e & b \geq e+2, c \geq e+2 \\ d-s-e-1 & b=e+1, c \geq e+2 \\ d-s-e-1 & b \geq e+2, c=e+1 \\ d-s-e-2 & b=c=e+1\end{cases}
$$

Case (i). We have $F_{d-s-e-1}=L_{e+1}^{1} F_{d-s-e-2}$, and since $F_{d-s-e-2}$ passes through all $d-s-e-1$ points of $\tilde{A}_{I}^{(e, 1)}$ we contradicts the minimality of $I$.

Case (ii). We assume $b=e+1$. Let us consider $\tilde{\tilde{A}}_{I}$ as we did before and we examine

$$
\#\left(F_{d-s-e-1} \cap \tilde{\tilde{A}}_{I}^{(0,2)}\right)= \begin{cases}d-s-e+1 & d-s-1 \geq c, 1 \leq d-s-e-r \\ d-s-e & c=d-s, 1 \leq d-s-e-r \\ d-s-e & d-s-1 \geq c, d-s-e-r=0 \\ d-s-e-1 & c=d-s, d-s-e-r=0\end{cases}
$$

In the second and third possibilities we obtain directly a contradiction with the minimality of $I$. In the last possibility we also obtain a contradiction. In fact, if $c=d-s$ and $s+r=d-e$, we do not remove any point of $H_{1}^{2}$ and we have $\#\left(F_{d-s-e-1} \cap \tilde{\tilde{A}}_{I}^{(1,2)}\right)=d-s-e$. Then $F_{d-s-e-1}=L_{1}^{2} F_{d-s-e-2}=L_{1}^{2} L_{0}^{2} F_{d-s-e-3}$, which contradicts the minimality of $I$.

Therefore if $b=e+1$, it must be $d-s-1 \geq c \geq e+2$ and $1 \leq d-s-e-r$. Iterating this argument we conclude that either $c=e+2$ and $r=2$ or $c=e+3$ and $r=3$. Therefore, either $I=\left(x_{0}^{d}, x_{1}^{d}, x_{2}^{d}\right)+$ $x_{0}^{s} x_{1}^{e} x_{2}^{d-s-e-2}\left(x_{0}^{2}, x_{0} x_{1}, x_{1}^{2}, x_{2}^{2}\right)$ which is of type (2); or $I=\left(x_{0}^{d}, x_{1}^{d}, x_{2}^{d}\right)+x_{0}^{s} x_{1}^{e} x_{2}^{d-s-e-3}\left(x_{0}^{3}, x_{0} x_{1} x_{2}, x_{1}^{3}, x_{2}^{3}\right)$ which is of type (3).

Case (iii). Arguing as in case (ii) we get $b=e+2$ and $r=2$. Therefore, $I=\left(x_{0}^{d}, x_{1}^{d}, x_{2}^{d}\right)+$ $x_{0}^{s} x_{1}^{e} x_{2}^{d-s-e-2}\left(x_{0}^{2}, x_{1} x, x_{1}^{2}, x_{2}^{2}\right)$ which is of type (2).

Case (iv). Arguing as in case (ii) we get that $r=2$ and $I$ is of type (1).

CASE d2: In this case we assume $e=0$ and $a \geq 1$. We will separate the case $b=1$ from the case $b \geq 2$.

If $b=1 \geq\left\lfloor\frac{d-s-1}{2}\right\rfloor$ then $s=d-3$ and $r=2$. Hence, $I=\left(x_{0}^{d}, x_{1}^{d}, x_{2}^{d}\right)+x_{0}^{d-3}\left(x_{0} x_{1} x_{2}, x_{1}^{3}, x_{2}^{3}\right)+$ $\left(x_{0}^{d-3} x_{0}^{2} x_{1}\right),\left(x_{0}^{d}, x_{1}^{d}, x_{2}^{d}\right)+x_{0}^{d-3}\left(x_{0}^{2} x_{1}, x_{0} x_{1} x_{2}, x_{1}^{2} x_{2}, x_{2}^{3}\right)$ or $\left(x_{0}^{d}, x_{1}^{d}, x_{2}^{d}\right)+x_{0}^{d-3}\left(x_{0}^{2} x_{1}, x_{0} x_{1} x_{2}, x_{1} x_{2}^{2}, x_{2}^{3}\right)$. The first one is not minimal and the remaining two are of type (4). 
Assume $b \geq 2$. Let us first suppose $d-s-r-1 \geq 0$ (i.e. $m_{1} \notin H_{0}^{2}$ ) and we consider

$$
\#\left(F_{d-s-1} \cap \tilde{A}_{I}^{(1,1)}\right)= \begin{cases}d-s & a \geq 2, c \geq 2 \\ d-s-1 & a=1, c \geq 2 \\ d-s-1 & a \geq 2, c=1 \\ d-s-2 & a=c=1\end{cases}
$$

Case (i). We get $F_{d-s-1}=L_{1}^{1} F_{d-s-2}=L_{0}^{1} L_{1}^{1} F_{d-s-3}$ which contradicts the minimality of $I$.

Case (ii). Assume that $a=1$ and $c \geq 2$. Suppose that $d-s-r-1>0$ and let us consider

$$
\#\left(F_{d-s-1} \cap \tilde{A}_{I}^{(0,2)}\right)= \begin{cases}d-s & d-s-2 \geq b, d-s-1 \geq c \\ d-s-1 & b=d-s-1, d-s-1 \geq c \\ d-s-1 & d-s-2 \geq b, c=d-s \\ d-s-2 & b=d-s-1, c=d-s .\end{cases}
$$

The first possibility contradicts the minimality of $I$.

Now let us suppose that $d-s-r-1>1$. In this case, the second (resp. third) possibility can occur if, and only if $b=c=d-s-1$ (resp. $b=d-s-2$ and $c=d-s$ ). Therefore $I$ is one of the next types:

$I=\left(x_{0}^{d}, x_{1}^{d}, x_{2}^{d}\right)+x_{0}^{s}\left(x_{0}^{r} x_{1} x_{2}^{d-s-r-1}, x_{0} x_{1}^{d-s-1}, x_{1}^{d-s-1} x_{2}, x_{2}^{d-s}\right)$ which does not correspond to a Togliatti system.

$I=\left(x_{0}^{d}, x_{1}^{d}, x_{2}^{d}\right)+x_{0}^{s}\left(x_{0}^{r} x_{1} x_{2}^{d-s-r-1}, x_{0} x_{1}^{d-s-2} x_{2}, x_{1}^{d-s}, x_{2}^{d-s}\right)$ which is a Togliatti system if, and only if $r=2$ and $s=d-5$ (of type (6)).

If $d-s-r-1=1$, then there are no special restrictions for the second and third case. Therefore $I$ is one of the next types:

$I=\left(x_{0}^{d}, x_{1}^{d}, x_{2}^{d}\right)+x_{0}^{s}\left(x_{0}^{d-s-2} x_{1} x_{2}, x_{0} x_{1}^{d-s-1}, x_{1}^{c} x_{2}^{d-s-c}, x_{2}^{d-s}\right)$ which is a Togliatti system if, and only if $s=d-4$ and $c=3$ (of type (5)), or

$I=\left(x_{0}^{d}, x_{1}^{d}, x_{2}^{d}\right)+x_{0}^{s}\left(x_{0}^{d-s-2} x_{1} x_{2}, x_{0} x_{1}^{b} x_{2}^{d-s-b-1}, x_{1}^{d-s}, x_{2}^{d-s}\right)$ which is a Togliatti system if, and only if $s=d-5$ and $b=2$ (of type (6)), or

$I=\left(x_{0}^{d}, x_{1}^{d}, x_{2}^{d}\right)+x_{0}^{s}\left(x_{0}^{r} x_{1} x_{2}^{d-s-r-1}, x_{0} x_{1}^{d-s-1}, x_{1}^{d-s}, x_{2}^{d-s}\right)$ which is a Togliatti system if, and only if $r=2$ and $s=d-3$ (of type (3)).

Now, let us suppose that $d-s-r-1=0$. Arguing as usual, we see that it cannot be $d-s-2 \geq c$ and $d-s-3 \geq b$. Therefore $d-s \geq c \geq d-s-1$ or $d-s-1 \geq b \geq d-s-2$, and we have the following possibilities:

$b=d-s-2, d-s-2 \geq c$ and $I=\left(x_{0}^{d}, x_{1}^{d}, x_{2}^{d}\right)+x_{0}^{s}\left(x_{0}^{d-s-1} x_{1}, x_{0} x_{1}^{d-s-2} x_{2}, x_{1}^{c} x_{2}^{d-s-c}, x_{2}^{d-s}\right)$ which is a Togliatti system if, and only if $s=d-3$ and $c=d-s-2$ (of type (4)).

$d-s-3 \geq b, c=d-s-1$ and $I=\left(x_{0}^{d}, x_{1}^{d}, x_{2}^{d}\right)+x_{0}^{s}\left(x_{0}^{d-s-1} x_{1}, x_{0} x_{1}^{b} x_{2}^{d-s-b-1}, x_{1}^{d-s-1} x_{2}, x_{2}^{d-s}\right)$ which is a Togliatti system if, and only if $d-3 \geq s \geq d-4$ and $b=d-s-3$ (resp. of type (4) and (5)).

$b=d-s-2, c=d-s-1$ and $I=\left(x_{0}^{d}, x_{1}^{d}, x_{2}^{d}\right)+x_{0}^{s}\left(x_{0}^{d-s-1} x_{1}, x_{0} x_{1}^{d-s-2} x_{2}, x_{1}^{d-s-1} x_{2}, x_{2}^{d-s}\right)$ which is a Togliatti system if, and only if $s=d-3$ (of type (4)).

$b=d-s-1, d-s-1 \geq c$ and $I=\left(x_{0}^{d}, x_{1}^{d}, x_{2}^{d}\right)+x_{0}^{s}\left(x_{0}^{d-s-1} x_{1}, x_{0} x_{1}^{d-s-1}, x_{1}^{c} x_{2}^{d-s-c}, x_{2}^{d-s}\right)$ which is a Togliatti system if, and only if $s=d-3$ and $d-s-1 \geq c \geq d-s-2$ (of type (4)).

$d-s-2 \geq b, c=d-s$ and $I=\left(x_{0}^{d}, x_{1}^{d}, x_{2}^{d}\right)+x_{0}^{s}\left(x_{0}^{d-s-1} x_{1}, x_{0} x_{1}^{b} x_{2}^{d-s-b-1}, x_{1}^{d-s}, x_{2}^{d-s}\right)$ which is a Togliatti system if, and only if $s=d-3$ and $d-s-2 \geq b \geq d-s-3$ (of type (4)).

$b=d-s-1, c=d-s$ and $I=\left(x_{0}^{d}, x_{1}^{d}, x_{2}^{d}\right)+x_{0}^{s}\left(x_{0}^{d-s-1} x_{1}, x_{0} x_{1}^{d-s-1}, x_{1}^{d-s}, x_{2}^{d-s}\right)$ which is a Togliatti system if, and only if $s=d-3$ (of type (4)). 
Case (iii). Assume $c=1$ and $a \geq 2$. We consider $\#\left(F_{d-s-1} \cap \tilde{A}_{I}^{(0,2)}\right)$ and we obtain that $I$ is one of the next types:

$I=\left(x_{0}^{d}, x_{1}^{d}, x_{2}^{d}\right)+x_{0}^{s}\left(x_{0}^{r} x_{1}^{d-s-r-1} x_{2}, x_{0} x_{1}^{d-s-1}, x_{1} x_{2}^{d-s-1}, x_{2}^{d-s}\right)$ which is a Togliatti system if, and only if $r=2$ and $d-3 \geq s \geq d-4$ (of type (4) and (5)).

$I=\left(x_{0}^{d}, x_{1}^{d}, x_{2}^{d}\right)+x_{0}^{s}\left(x_{0}^{r} x_{1}^{d-s-r}, x_{0} x_{1}^{d-s-2} x_{2}, x_{1} x_{2}^{d-s-1}, x_{2}^{d-s}\right)$ which is a Togliatti system if, and only if $r=2$ and $s=d-3$ (of type (4)).

$I=\left(x_{0}^{d}, x_{1}^{d}, x_{2}^{d}\right)+x_{0}^{s}\left(x_{0}^{r} x_{1}^{d-s-r}, x_{0} x_{1}^{d-s-1}, x_{1} x_{2}^{d-s-1}, x_{2}^{d-s}\right)$. In this case, let us consider \# $\left(F_{d-s-1} \cap\right.$ $\left.\tilde{A}_{I}^{(1,2)}\right)=d-s$ and inductively we obtain $F_{d-s-1}=L_{1}^{2} \cdots L_{d-s-2}^{1} F_{1}$. Therefore it must be $r=2$ and $s=d-3$, and $I$ is of type (4).

Case (iv). Assume $a=c=1$. Let us first suppose that $d-s-r-1>0$. If $d-s-2 \geq b$ we factorize $F_{d-s-1}$ as $F_{d-s-1}=L_{0}^{2} F_{d-s-2}$ which contradicts the minimality of $I$. Therefore, $b=d-s-1$ and we factorize $F_{d-s-1}=L_{1}^{2} \cdots L_{d-s-r-2} F_{r+1}$. Since \# $\left(F_{r} \cap \tilde{A}_{I}^{(0,2)}\right)=d-s-1$ we have $r+1 \geq d-s-1$ and then $d-s-$ $r-1 \leq 1$. Therefore, $d-s-r-1=1$ and $I=\left(x_{0}^{d}, x_{1}^{d}, x_{2}^{d}\right)+x_{0}^{s}\left(x_{0}^{d-s-2} x_{1} x_{2}, x_{0} x_{1}^{d-s-1}, x_{1} x_{2}^{d-s-1}, x_{2}^{d-s}\right)$. It is a Togliatti system if, and only if $s=d-4$ (of type (5)).

If $d-s-r-1=0$, we use the same argumentation to prove that $d-s-1 \geq b \geq d-s-2$ and then we have two possibilities:

$$
\begin{aligned}
& b=d-s-1 \text { and } I=\left(x_{0}^{d}, x_{1}^{d}, x_{2}^{d}\right)+x_{0}^{s}\left(x_{0}^{d-s-1} x_{1}, x_{0} x_{1}^{d-s-1}, x_{1} x_{2}^{d-s-1}, x_{2}^{d-s}\right) \\
& b=d-s-2 \text { and } I=\left(x_{0}^{d}, x_{1}^{d}, x_{2}^{d}\right)+x_{0}^{s}\left(x_{0}^{d-s-1} x_{1}, x_{0} x_{1}^{d-s-2} x_{2}, x_{1} x_{2}^{d-s-1}, x_{2}^{d-s}\right)
\end{aligned}
$$

They are Togliatti systems if, and only if $s=d-3$ (of type (4)).

CASE d3: Let us assume $e \geq 1$ and $a=0$. Actually, it must be $e=1$. Otherwise, $e>1$ and $\#\left(F_{d-s-1} \cap\right.$ $\left.\tilde{A}_{I}^{(1,1)}\right)=d-s$, and we have seen that this cannot happen.

Now, let us suppose $d-s-r>1$. Arguing as before we see that there are three possibilities:

$b=d-s-1, c=d-s-1$ and $I=\left(x_{0}^{d}, x_{1}^{d}, x_{2}^{d}\right)+x_{0}^{s}\left(x_{0}^{r} x_{2}^{d-s-r}, x_{0} x_{1}^{d-s-1}, x_{1}^{d-s-1} x_{2}, x_{1} x_{2}^{d-s-1}\right)$.

$b=d-s-2, c=d-s$ and $\mathrm{a} I=\left(x_{0}^{d}, x_{1}^{d}, x_{2}^{d}\right)+x_{0}^{s}\left(x_{0}^{r} x_{2}^{d-s-r}, x_{0} x_{1}^{d-s-2} x_{2}, x_{1}^{d-s}, x_{1} x_{2}^{d-s-1}\right)$.

They do not correspond to a Togliatti system.

$b=d-s-1, c=d-s$. If $d-2>s+r$, then we have the factorization $F_{d-s-1}=L_{1}^{2} \cdots L_{d-s-r-1} F_{r}$ and $\#\left(F_{r} \cap \tilde{A}_{I}^{(0,2)}\right)=d-s-2>r$, which contradicts the minimality of $I$. Hence we have $s+r=d-2$ and $I=\left(x_{0}^{d}, x_{1}^{d}, x_{2}^{d}\right)+x_{0}^{s}\left(x_{0}^{d-s-2} x_{2}^{2}, x_{0} x_{1}^{d-s-1}, x_{1}^{d-s}, x_{1} x_{2}^{d-s-1}\right)$ which is never a Togliatti system since $s \leq d-4$.

To finish, assume $d-s-r=1$. Arguing in the same manner, we see that it cannot occur $d-s-3 \geq b$ and $d-s-2 \geq c \geq 1$. Therefore we have the following possibilities:

$d-s-3 \geq b, c=d-s-1$ and $I=\left(x_{0}^{d}, x_{1}^{d}, x_{2}^{d}\right)+x_{0}^{s}\left(x_{0}^{d-s-1} x_{2}, x_{0} x_{1}^{b} x_{2}^{d-s-b-1}, x_{1}^{d-s-1} x_{2}, x_{1} x_{2}^{d-s-1}\right)$, it is a Togliatti system if, and only if $s=d-3$ and $b=d-s-3$ (of type (4)).

$b=d-s-2, c=d-s-1$ and $I=\left(x_{0}^{d}, x_{1}^{d}, x_{2}^{d}\right)+x_{0}^{s}\left(x_{0}^{d-s-1} x_{2}, x_{0} x_{1}^{d-s-2} x_{2}, x_{1}^{d-s-1} x_{2}, x_{1} x_{2}^{d-s-1}\right)$, it is a Togliatti system if, and only if $s=d-3$ but it is not minimal.

$b=d-s-1, d-s-1 \geq c$ and $I=\left(x_{0}^{d}, x_{1}^{d}, x_{2}^{d}\right)+x_{0}^{s}\left(x_{0}^{d-s-1} x_{2}, x_{0} x_{1}^{d-s-1}, x_{1}^{c} x_{2}^{d-s-c}, x_{1} x_{2}^{d-s-1}\right)$, it is a Togliatti system if, and only if $s=d-3$ and $c=d-s-1$ (of type (4)).

$d-s-2 \geq b, c=d-s$ and $I=\left(x_{0}^{d}, x_{1}^{d}, x_{2}^{d}\right)+x_{0}^{s}\left(x_{0}^{d-s-1} x_{2}, x_{0} x_{1}^{b} x_{2}^{d-s-b-1}, x_{1}^{d-s}, x_{1} x_{2}^{d-s-1}\right)$. It is a Togliatti system if, and only if $s=d-3$ and $d-s-2 \geq b \geq d-s-3$ (of type (4)), or $s=d-4$ and $b=d-s-2($ of type (5)).

$b=d-s-1, c=d-s$ and $I=\left(x_{0}^{d}, x_{1}^{d}, x_{2}^{d}\right)+x_{0}^{s}\left(x_{0}^{d-s-1} x_{2}, x_{0} x_{1}^{d-s-2} x_{2}, x_{1}^{d-s}, x_{1} x_{2}^{d-s-1}\right)$, it is a Togliatti system if, and only if $s=d-3$ (of type (4)).

CASE d4: Let us assume $e=a=0$. If $b \geq 3$ and $c \geq 3$, we have the factorization $F_{d-s-1}=L_{1}^{1} L_{2}^{1} F_{d-s-3}$ and $\#\left(F_{d-s-3} \cap \tilde{A}_{I}^{(0,1)}\right)=d-s-2$ and we contradict the minimality of $I$. Hence we distinguish three cases: $b=1, b=2$ and $b \geq 3$. 
Case (i). We assume $b=1$. Since $b \geq\left\lfloor\frac{d-s-1}{2}\right\rfloor$ it must be $s=d-3$. Therefore $I=$ $\left.x_{0}^{d}, x_{1}^{d}, x_{2}^{d}\right)+x_{0}^{d-3}\left(x_{0} x_{1} x_{2}, x_{1}^{3}, x_{2}^{3}\right)+\left(x_{0}^{d-1} x_{2}\right),\left(x_{0}^{d}, x_{1}^{d}, x_{2}^{d}\right)+x_{0}^{d-3}\left(x_{0}^{2} x_{2}, x_{0} x_{1} x_{2}, x_{1}^{2} x_{2}, x_{2}^{3}\right)$ or $\left(x_{0}^{d}, x_{1}^{d}, x_{2}^{d}\right)+$ $x_{0}^{d-3}\left(x_{0}^{2} x_{2}, x_{0} x_{1} x_{2}, x_{1} x_{2}^{2}, x_{2}^{3}\right)$. The first one is not minimal while the remaining two are of type (1).

Case (ii). We assume $b=2$. Since $b \geq\left\lfloor\frac{d-s-1}{2}\right\rfloor$ it must be $d-3 \geq s \geq d-5$.

If $s=d-3, I=\left(x_{0}^{d}, x_{1}^{d}, x_{2}^{d}\right)+x_{0}^{d-3}\left(x_{0}^{2} x_{2}, x_{0} x_{1}^{2}, x_{1}^{3}, x_{2}^{3}\right),\left(x_{0}^{d}, x_{1}^{d}, x_{2}^{d}\right)+x_{0}^{d-3}\left(x_{0}^{2} x_{2}, x_{0} x_{1}^{2}, x_{1}^{2} x_{2}, x_{2}^{3}\right)$ or $\left(x_{0}^{d}, x_{1}^{d}, x_{2}^{d}\right)+x_{0}^{d-3}\left(x_{0}^{2} x_{2}, x_{0} x_{1}^{2}, x_{1} x_{2}^{2}, x_{2}^{3}\right)$. All of them are of type (4).

If $s=d-4, I=\left(x_{0}^{d}, x_{1}^{d}, x_{2}^{d}\right)+x_{0}^{d-4}\left(x_{0}^{3} x_{2}, x_{0} x_{1}^{2} x_{2}, x_{1}^{4}, x_{2}^{4}\right),\left(x_{0}^{d}, x_{1}^{d}, x_{2}^{d}\right)+x_{0}^{d-4}\left(x_{0}^{3} x_{2}, x_{0} x_{1}^{2} x_{2}\right.$, $\left.x_{1}^{3} x_{2}, x_{2}^{4}\right),\left(x_{0}^{d}, x_{1}^{d}, x_{2}^{d}\right)+x_{0}^{d-4}\left(x_{0}^{3} x_{2}, x_{0} x_{1}^{2} x_{2}, x_{1}^{2} x_{2}^{2}, x_{2}^{4}\right),\left(x_{0}^{d}, x_{1}^{d}, x_{2}^{d}\right)+x_{0}^{d-4}\left(x_{0}^{3} x_{2}, x_{0} x_{1}^{2} x_{2}, x_{1} x_{2}^{3}, x_{2}^{4}\right)$, $\left(x_{0}^{d}, x_{1}^{d}, x_{2}^{d}\right)+x_{0}^{d-4}\left(x_{0}^{2} x_{2}^{2}, x_{0} x_{1}^{2} x_{2}, x_{1}^{4}, x_{2}^{4}\right),\left(x_{0}^{d}, x_{1}^{d}, x_{2}^{d}\right)+x_{0}^{d-4}\left(x_{0}^{2} x_{2}^{2}, x_{0} x_{1}^{2} x_{2}, x_{1}^{3} x_{2}, x_{2}^{4}\right),\left(x_{0}^{d}, x_{1}^{d}, x_{2}^{d}\right)+$ $x_{0}^{d-4}\left(x_{0}^{2} x_{2}^{2}, x_{0} x_{1}^{2} x_{2}, x_{1}^{2} x_{2}^{2}, x_{2}^{4}\right),\left(x_{0}^{d}, x_{1}^{d}, x_{2}^{d}\right)+x_{0}^{d-4}\left(x_{0}^{2} x_{2}^{2}, x_{0} x_{1}^{2} x_{2}, x_{1} x_{2}^{3}, x_{2}^{4}\right)$. Only the fifth one is a minimal Togliatti system, and it is of type (5).

Finally, if $s=d-5, I$ has 15 possibilities, but any of them is a minimal Togliatti system.

Case (iii). We assume $b \geq 3$. Then, either $c=1$ or $c=2$.

Case $c=1$. We will see that $b=d-s-1$. Suppose $d-s-2 \geq b \geq 3$, then $\#\left(F_{d-s-1} \cap \tilde{A}_{I}^{(0,2)}\right)=d-s$ and $F_{d-s-1}=L_{0}^{2} F_{d-s-2}$. First we will see that this implies that $m_{1}$ and $m_{2}$ are aligned vertically (i.e. $d-s-b-1=d-s-r)$. We suppose that $d-s-b-1 \leq d-s-r$, and then $b \geq r-1$ (the other case is symmetric). Inductively we obtain $F_{d-s-1}=L_{0}^{2} L_{1}^{2} \cdots L_{d-s-b-2}^{2} F_{b}$. If $b>r-1$, then $\# \tilde{A}_{I}^{(d-s-b-1,2)}=b+1$ and it would mean to a contradiction with the minimality of $I$. Hence, $b=r-1$ and we get the factorization $F_{d-s-1}=L_{0}^{2} L_{1}^{2} \cdots L_{d-s-b-2}^{2} L_{d-s-b}^{2} \cdots L_{d-s-2} F_{1}$. Since \# $\tilde{A}_{I}^{(d-s-b-1,2)}=$ $b \geq 3$ we have again a contradiction with the minimality.

Once we have seen that $b=d-s-1$, using the usual argumentation we see that $d-s-r=1$. Therefore $I=\left(x_{0}^{d}, x_{1}^{d}, x_{2}^{d}\right)+x_{0}^{s}\left(x_{0}^{d-s-1} x_{2}, x_{0} x_{1}^{d-s-1}, x_{1} x_{2}^{d-s-1}, x_{2}^{d-s}\right)$ with $s \leq d-3$, which is Togliatti if, and only if $s=d-3$ and it is of type (4).

Case $c=2$. Since $\# \tilde{A}_{I}^{(1,1)}=d-s$ we have $F_{d-s-1}=L_{1}^{1} F_{d-s-2}$. If $d-s-2 \geq b \geq 3$, then $\# \tilde{\tilde{A}}_{I}^{(0,2)}=d-s-1$ and $F_{d-s-1}$ would factorize as $F_{d-s-1}=L_{1}^{1} L_{0}^{2} F_{d-s-3}$. This contradicts the minimality of $I$ because $\# \tilde{\tilde{A}}_{I}^{(0,1)}=d-s-2$ which would force the factorization $F_{d-s-1}=$ $L_{1}^{1} L_{0}^{2} L_{0}^{1} F_{d-s-4}$. Therefore $b=d-s-1$ and again by minimality we see that $d-s-r=1$. Hence, $I=\left(x_{0}^{d}, x_{1}^{d}, x_{2}^{d}\right)+x_{0}^{s}\left(x_{0}^{d-s-1} x_{2}, x_{0} x_{1}^{d-s-1}, x_{1}^{2} x_{2}^{d-s-2}, x_{2}^{d-s}\right)$, which is Togliatti if, and only if $s=d-3$ and in this case it is of type (4)

To finish case 2D we see what happens when $d-s \geq c>e \geq\left\lfloor\frac{d-s-1}{2}\right\rfloor$. We see using the minimality that either $a \geq b=e, b \geq a=e$ or $e \geq a=b$.

Arguing as before we see that in the first possibility $m_{1}$ and $m_{3}$ must be vertically aligned and in particular $c=e+2, a=e$ and $r=2$. Therefore $I=\left(x_{0}^{d}, x_{1}^{d}, x_{2}^{d}\right)+x_{0}^{s} x_{1}^{e} x_{2}^{d-s-e-2}\left(x_{0}^{2}, x_{0} x_{1}, x_{1}^{2}, x_{2}^{2}\right)$ which is of type (1).

Now we assume $b \geq a=e$. If $b, c \geq e+1$, then we have the factorization $F_{d-s-e-1}=L_{e+1}^{1} F_{d-s-e-2}$ and, since $\# \tilde{\tilde{A}}_{I}^{(e, 1)}=d-s-e-1$ we get $F_{d-s-e-1}=L_{e}^{1} L_{e+1}^{1} F_{d-s-e-3}$ which contradicts the minimality. Now, suppose $b=e+1$ and $c \geq e+2$ (resp. $b \geq e+2$ and $c=e+1$ ). As we have seen earlier, $m_{1}$ and $m_{3}$ (resp. $m_{2}$ ) must be aligned. Therefore, we can see using the minimality assumption that $r=2$ and $c=e+2$ (resp. $r=2$ and $b=e+2$ ). In both cases $I$ is of type (1).

Finally, let us assume $e \geq a=b$. If $e \geq a+2$, we get a contradiction with the minimality of $I$. Hence either $e=a$ or $e=a+1$. If $e=a$ we see that $c=a+2$ and $r=2$. Therefore $I$ is of type (1). Otherwise $e=a+1$ and we get $c=a+2$ and $r=2$ and $I$ is of type (1).

For any integer $d \geq 3$, set $M^{0}(d)=\left\{x_{0}^{a} x_{1}^{b} x_{2}^{c} \mid a+b+c=d\right.$ and $\left.a, b, c \geq 1\right\}$. 
Theorem 3.9. Let $I \subset k\left[x_{0}, \ldots, x_{n}\right]$ be a smooth minimal monomial Togliatti system of forms of degree $d \geq 10$. Assume that $\mu(I)=2 n+3$. Then, $n=2$ and, up to a permutation of the coordinates, one of the following cases holds:

(i) $I=\left(x_{0}^{d}, x_{1}^{d}, x_{2}^{d}\right)+m\left(x_{0}^{2}, x_{1}^{2}, x_{0} x_{2}, x_{1} x_{2}\right)$ with $m \in M^{0}(d-2)$, or

(ii) $I=\left(x_{0}^{d}, x_{1}^{d}, x_{2}^{d}\right)+m\left(x_{0}^{2}, x_{1}^{2}, x_{0} x_{1}, x_{2}^{2}\right)$ with $m \in M^{0}(d-2)$, or

(iii) $I=\left(x_{0}^{d}, x_{1}^{d}, x_{2}^{d}\right)+m\left(x_{0}^{3}, x_{1}^{3}, x_{2}^{3}, x_{0} x_{1} x_{2}\right)$ with $m \in M^{0}(d-3)$.

Proof. By [4, Proposition 4.1], for $n \geq 3$ and $d \geq 4$ there are no smooth minimal monomial Togliatti systems $I \subset k\left[x_{0}, \ldots, x_{n}\right]$ of forms of degree $d$ with $\mu(I)=2 n+3$. So, $n=2$. For $n=2$, the result follows from Theorem 3.8 together with the smoothness criterion Proposition 3.6.

The following remarks shows that in the above Theorem the hypothesis of being smooth cannot be deleted.

Remark 3.10. If $n=3$ and $d \geq 10$ one can easily check that $I=\left(x_{0}^{d}, x_{1}^{d}, x_{2}^{d}, x_{3}^{d}\right)+x_{0}^{d-2}\left(x_{0} x_{1}, x_{2} x_{3}, x_{1}^{2}, x_{2}^{2}, x_{3}^{2}\right)$ is a minimal monomial Togliatti systems of forms of degree $d$ with $\mu(I)=2 n+3=9$ and it is nonsmooth.

Remark 3.11. For $n=2$ and $6 \leq d \leq 9$ one can check with the help of Macaulay2 [3] that there exist other examples of minimal monomial Togliatti systems $I=\left(x_{0}^{d}, x_{1}^{d}, x_{2}^{d}\right)+J \subset k\left[x_{0}, x_{1}, x_{2}\right]$ with $\mu(I)=7$. For seek of completeness we give the full list of possible J's not included in Theorem 3.8:

$\underline{d=6}:\left(x_{0}^{5} x_{1}, x_{0}^{3} x_{2}^{3}, x_{0}^{2} x_{1}^{3} x_{2}, x_{1}^{5} x_{2}\right),\left(x_{0}^{5} x_{2}, x_{0}^{3} x_{1}^{3}, x_{0}^{2} x_{1}^{2} x_{2}^{2}, x_{1}^{5} x_{2}\right),\left(x_{0}^{3} x_{2}^{3}, x_{0}^{2} x_{1}^{4}, x_{0}^{2} x_{1}^{2} x_{2}^{2}, x_{1}^{5} x_{2}\right),\left(x_{0}^{5} x_{2}, x_{0}^{3} x_{1}^{3}\right.$, $\left.x_{0} x_{1} x_{2}^{4}, x_{1}^{5} x_{2}\right),\left(x_{0}^{4} x_{2}^{2}, x_{0}^{3} x_{1}^{3}, x_{0}^{2} x_{1}^{2} x_{2}^{2}, x_{1}^{4} x_{2}^{2}\right),\left(x_{0}^{3} x_{2}^{3}, x_{0}^{2} x_{1}^{4}, x_{0}^{2} x_{1}^{2} x_{2}^{2}, x_{1}^{4} x_{2}^{2}\right),\left(x_{0}^{4} x_{2}^{2}, x_{0}^{3} x_{1}^{3}, x_{0} x_{1} x_{2}^{4}, x_{1}^{4} x_{2}^{2}\right),\left(x_{0}^{3} x_{1}^{34}\right.$, $\left.x_{0}^{3} x_{2}^{3}, x_{0}^{2} x_{1}^{2} x_{2}^{2}, x_{1}^{3} x_{2}^{3}\right), x_{0} x_{1}\left(x_{0}^{4}, x_{0}^{2} x_{1}^{2}, x_{0} x_{1} x_{2}^{2}, x_{1}^{4}\right), x_{0} x_{1}\left(x_{0}^{3} x_{2}, x_{0}^{2} x_{1}^{2}, x_{0} x_{1} x_{2}^{2}, x_{1}^{3} x_{2}\right), x_{0} x_{1}\left(x_{0}^{2} x_{1}^{2}, x_{0}^{2} x_{2}^{2}, x_{0} x_{1} x_{2}^{2}\right.$, $\left.x_{1}^{2} x_{2}^{2}\right), x_{0} x_{1}\left(x_{0}^{2} x_{1}^{2}, x_{0}^{2} x_{2}^{2}, x_{0} x_{2}^{3}, x_{1}^{2} x_{2}^{2}\right), x_{0} x_{1}\left(x_{0}^{4}, x_{0} x_{2}^{3}, x_{1}^{4}, x_{1}^{2} x_{2}^{2}\right), x_{0} x_{1}\left(x_{0}^{4}, x_{0}^{2} x_{1}^{2}, x_{1}^{4}, x_{2}^{4}\right), x_{0} x_{1}\left(x_{0}^{4}, x_{0} x_{1} x_{2}^{2}, x_{1}^{4}\right.$, $\left.x_{2}^{4}\right), x_{0} x_{1}\left(x_{0}^{3} x_{2}, x_{0}^{2} x_{1}^{2}, x_{1}^{3} x_{2}, x_{2}^{4}\right), x_{0} x_{2}\left(x_{0}^{3} x_{2}, x_{0}^{2} x_{2}^{2}, x_{0} x_{1} x_{2}^{2}, x_{1}^{4}\right), x_{0} x_{2}\left(x_{0}^{2} x_{1} x_{2}, x_{0}^{2} x_{2}^{2}, x_{0} x_{1} x_{2}^{2}, x_{1}^{4}\right), x_{0} x_{2}\left(x_{0}^{3} x_{2}\right.$, $\left.x_{0} x_{1}^{2} x_{2}, x_{0} x_{1} x_{2}^{2}, x_{1}^{4}\right), x_{0} x_{2}\left(x_{0}^{3} x_{2}, x_{0}^{2} x_{1} x_{2}, x_{0} x_{2}^{3}, x_{1}^{4}\right), x_{0} x_{2}\left(x_{0}^{3} x_{2}, x_{0}^{2} x_{2}^{2}, x_{0} x_{2}^{3}, x_{1}^{4}\right), x_{0} x_{2}\left(x_{0}^{3} x_{2}, x_{0} x_{1}^{2} x_{2}, x_{0} x_{2}^{3}, x_{1}^{4}\right)$, $x_{0} x_{2}\left(x_{0}^{2} x_{1}^{2}, x_{0}^{2} x_{2}^{2}, x_{0} x_{1}^{3}, x_{1}^{3} x_{2}\right), x_{0} x_{2}\left(x_{0}^{2} x_{1}^{2}, x_{0}^{2} x_{2}^{2}, x_{0} x_{1}^{2} x_{2}, x_{1}^{3} x_{2}\right), x_{0} x_{2}\left(x_{0}^{2} x_{2}^{2}, x_{0} x_{1}^{3}, x_{0} x_{1}^{2} x_{2}, x_{1}^{3} x_{2}\right), x_{0} x_{2}\left(x_{0}^{2} x_{1}^{2}\right.$, $\left.x_{0}^{2} x_{2}^{2}, x_{0} x_{1}^{3}, x_{1}^{4}\right), x_{0} x_{2}\left(x_{0}^{2} x_{1}^{2}, x_{0}^{2} x_{2}^{2}, x_{1}^{4}, x_{1}^{3} x_{2}\right), x_{0} x_{2}\left(x_{0}^{2} x_{1}^{2}, x_{0}^{2} x_{2}^{2}, x_{1}^{4}, x_{1}^{2} x_{2}^{2}\right), x_{0} x_{2}\left(x_{0}^{3} x_{2}, x_{0}^{2} x_{1} x_{2}, x_{0} x_{1}^{2} x_{2}, x_{1}^{4}\right)$, $x_{0} x_{2}\left(x_{0}^{3} x_{2}, x_{0}^{2} x_{1} x_{2}, x_{0} x_{1} x_{2}^{2}, x_{1}^{4}\right), x_{0}\left(x_{0} x_{1}^{4}, x_{0} x_{1} x_{2}^{3}, x_{0} x_{2}^{4}, x_{1}^{3} x_{2}^{2}\right), x_{0}\left(x_{0}^{4} x_{2}, x_{0}^{2} x_{1}^{3}, x_{0} x_{1}^{2} x_{2}^{2}, x_{1}^{5}\right), x_{0}\left(x_{0}^{4} x_{2}, x_{0} x_{1} x_{2}^{3}\right.$, $\left.x_{1}^{5}, x_{1}^{3} x_{2}^{2}\right), x_{0}\left(x_{0}^{2} x_{2}^{3}, x_{0} x_{1}^{4}, x_{0} x_{1}^{2} x_{2}^{2}, x_{1}^{5}\right), x_{0}\left(x_{0}^{4} x_{2}, x_{0}^{2} x_{1} x_{2}^{2}, x_{1}^{5}, x_{1}^{2} x_{2}^{3}\right), x_{0}\left(x_{0}^{2} x_{2}^{3}, x_{0} x_{1}^{4}, x_{0} x_{1} x_{2}^{3}, x_{1}^{3} x_{2}^{2}\right), x_{0}\left(x_{0}^{4} x_{2}\right.$, $\left.x_{0}^{2} x_{2}^{3}, x_{0} x_{1}^{3} x_{2}, x_{1}^{5}\right), x_{0}\left(x_{0}^{4} x_{2}, x_{0}^{2} x_{1}^{3}, x_{1}^{5}, x_{1} x_{2}^{4}\right), x_{0}\left(x_{0}^{3} x_{2}^{2}, x_{0}^{2} x_{1}^{3}, x_{0} x_{2}^{4}, x_{1}^{3} x_{2}^{2}\right), x_{0}\left(x_{0}^{4} x_{2}, x_{0} x_{1}^{2} x_{2}^{2}, x_{1}^{5}, x_{1} x_{2}^{4}\right), x_{0}\left(x_{0}^{2} x_{2}^{3}\right.$, $\left.x_{0} x_{1}^{4}, x_{0} x_{2}^{4}, x_{1}^{3} x_{2}^{2}\right), x_{0}\left(x_{0}^{2} x_{1}^{3}, x_{0}^{2} x_{2}^{3}, x_{1}^{4} x_{2}, x_{1} x_{2}^{4}\right), x_{0}\left(x_{0}^{4} x_{1}, x_{0}^{2} x_{2}^{3}, x_{0} x_{1}^{3} x_{2}, x_{1}^{5}\right), x_{0}\left(x_{0}^{2} x_{1} x_{2}^{2}, x_{0} x_{1}^{3} x_{2}, x_{1}^{5}, x_{2}^{5}\right)$

$\underline{d=7}: x_{0} x_{1}\left(x_{0}^{2} x_{2}^{3}, x_{0} x_{1}^{4}, x_{0} x_{1}^{2} x_{2}^{2}, x_{1}^{5}\right), x_{0} x_{1}\left(x_{0}^{5}, x_{0}^{2} x_{1}^{2} x_{2}, x_{0} x_{1} x_{2}^{3}, x_{1}^{5}\right), x_{0} x_{1}\left(x_{0}^{4} x_{1}, x_{0}^{3} x_{1}^{2}, x_{0} x_{2}^{4}, x_{1}^{3} x_{2}^{2}\right), x_{0} x_{1}\left(x_{0}^{3} x_{1}^{2}\right.$, $\left.x_{0}^{2} x_{1}^{3}, x_{0}^{2} x_{2}^{3}, x_{1}^{2} x_{2}^{3}\right), x_{0} x_{1}\left(x_{0}^{5}, x_{0}^{2} x_{1}^{2} x_{2}, x_{1}^{5}, x_{2}^{5}\right), x_{0} x_{1}\left(x_{0}^{4} x_{2}, x_{0} x_{1}^{4}, x_{1}^{5}, x_{2}^{5}\right), x_{0} x_{1}\left(x_{0}^{5}, x_{0} x_{1} x_{2}^{3}, x_{1}^{5}, x_{2}^{5}\right), x_{0} x_{2}\left(x_{0}^{3} x_{2}^{2}\right.$, $\left.x_{0}^{2} x_{2}^{3}, x_{0} x_{1}^{3} x_{2}, x_{1}^{5}\right), x_{0} x_{2}\left(x_{0}^{4} x_{2}, x_{0}^{2} x_{1} x_{2}^{2}, x_{0} x_{2}^{4}, x_{1}^{5}\right), x_{0} x_{2}\left(x_{0}^{4} x_{2}, x_{0} x_{1}^{3} x_{2}, x_{0} x_{2}^{4}, x_{1}^{5}\right), x_{0}\left(x_{0}^{5} x_{2}, x_{0}^{2} x_{1}^{3} x_{2}, x_{0} x_{1}^{2} x_{2}^{3}, x_{1}^{6}\right)$, $x_{0}\left(x_{0} x_{1}^{5}, x_{0} x_{1}^{2} x_{2}^{3}, x_{0} x_{2}^{5}, x_{1}^{4} x_{2}^{2}\right), x_{0}\left(x_{0}^{5} x_{2}, x_{0}^{4} x_{1}^{2}, x_{0}^{2} x_{1}^{2} x_{2}^{2}, x_{1}^{3} x_{2}^{3}\right), x_{0}\left(x_{0}^{4} x_{1}^{2}, x_{0}^{4} x_{2}^{2}, x_{0}^{2} x_{1}^{2} x_{2}^{2}, x_{1}^{3} x_{2}^{3}\right), x_{0}\left(x_{0}^{3} x_{1}^{3}, x_{0}^{3} x_{2}^{3}\right.$, $\left.x_{0}^{2} x_{1}^{2} x_{2}^{2}, x_{1}^{3} x_{2}^{3}\right), x_{0}\left(x_{0}^{4} x_{1} x_{2}, x_{0}^{2} x_{1}^{4}, x_{0}^{2} x_{2}^{4}, x_{1}^{3} x_{2}^{3}\right), x_{0}\left(x_{0}^{2} x_{1}^{4}, x_{0}^{2} x_{1}^{2} x_{2}^{2}, x_{0}^{2} x_{2}^{4}, x_{1}^{3} x_{2}^{3}\right), x_{0}\left(x_{0}^{4} x_{1} x_{2}, x_{0} x_{1}^{5}, x_{0} x_{2}^{5}, x_{1}^{3} x_{2}^{3}\right)$, $x_{0}\left(x_{0}^{2} x_{1}^{2} x_{2}^{2}, x_{0} x_{1}^{5}, x_{0} x_{2}^{5}, x_{1}^{3} x_{2}^{3}\right), x_{0}\left(x_{0}^{5} x_{2}, x_{0}^{2} x_{1}^{3} x_{2}, x_{1}^{6}, x_{1} x_{2}^{5}\right), x_{0}\left(x_{0}^{5} x_{2}, x_{0} x_{1}^{2} x_{2}^{3}, x_{1}^{6}, x_{1} x_{2}^{5}\right), x_{0}\left(x_{0}^{5} x_{2}, x_{0}^{4} x_{1}^{2}, x_{1}^{5} x_{2}\right.$, $\left.x_{1} x_{2}^{5}\right), x_{0}\left(x_{0}^{4} x_{1}^{2}, x_{0}^{4} x_{2}^{2}, x_{1}^{5} x_{2}, x_{1} x_{2}^{5}\right), x_{0}\left(x_{0}^{3} x_{1}^{3}, x_{0}^{3} x_{2}^{3}, x_{1}^{5} x_{2}, x_{1} x_{2}^{5}\right), x_{0}\left(x_{0}^{4} x_{1} x_{2}, x_{0}^{2} x_{1}^{2} x_{2}^{2}, x_{1}^{6}, x_{2}^{6}\right), x_{0}\left(x_{0}^{4} x_{1} x_{2}, x_{1}^{6}\right.$, $\left.x_{1}^{3} x_{2}^{3}, x_{2}^{6}\right), x_{0}\left(x_{0}^{2} x_{1}^{2} x_{2}^{2}, x_{1}^{6}, x_{1}^{3} x_{2}^{3}, x_{2}^{6}\right), x_{0} x_{1} x_{2}\left(x_{0}^{2} x_{1}^{2}, x_{0}^{2} x_{2}^{2}, x_{0} x_{1}^{3}, x_{1}^{4}\right), x_{0} x_{1} x_{2}\left(x_{0}^{3} x_{2}, x_{0}^{2} x_{1} x_{2}, x_{0} x_{1}^{2} x_{2}, x_{1}^{4}\right), x_{0} x_{1} x_{2}\left(x_{0}^{4}\right.$, $\left.x_{0}^{2} x_{1}^{2}, x_{0} x_{1} x_{2}^{2}, x_{1}^{4}\right), x_{0} x_{1} x_{2}\left(x_{0}^{3} x_{2}, x_{0}^{2} x_{1} x_{2}, x_{0} x_{1} x_{2}^{2}, x_{1}^{4}\right), x_{0} x_{1} x_{2}\left(x_{0}^{3} x_{2}, x_{0}^{2} x_{2}^{2}, x_{0} x_{1} x_{2}^{2}, x_{1}^{4}\right), x_{0} x_{1} x_{2}\left(x_{0}^{2} x_{1} x_{2}, x_{0}^{2} x_{2}^{2}\right.$, $\left.x_{0} x_{1} x_{2}^{2}, x_{1}^{4}\right), x_{0} x_{1} x_{2}\left(x_{0}^{3} x_{2}, x_{0} x_{1}^{2} x_{2}, x_{0} x_{1} x_{2}^{2}, x_{1}^{4}\right), x_{0} x_{1} x_{2}\left(x_{0}^{3} x_{2}, x_{0}^{2} x_{1} x_{2}, x_{0} x_{2}^{3}, x_{1}^{4}\right), x_{0} x_{1} x_{2}\left(x_{0}^{3} x_{2}, x_{0}^{2} x_{2}^{2}, x_{0} x_{2}^{3}, x_{1}^{4}\right)$, $x_{0} x_{1} x_{2}\left(x_{0}^{3} x_{1}, x_{0} x_{1}^{3}, x_{0} x_{2}^{3}, x_{1}^{4}\right), x_{0} x_{1} x_{2}\left(x_{0}^{3} x_{2}, x_{0} x_{1}^{2} x_{2}, x_{0} x_{2}^{3}, x_{1}^{4}\right), x_{0} x_{1} x_{2}\left(x_{0}^{2} x_{1}^{2}, x_{0}^{2} x_{2}^{2}, x_{0} x_{1}^{3}, x_{1}^{3} x_{2}\right), x_{0} x_{1} x_{2}\left(x_{0}^{2} x_{1}^{2}\right.$, $\left.x_{0}^{2} x_{2}^{2}, x_{0} x_{1}^{2} x_{2}, x_{1}^{3} x_{2}\right), x_{0} x_{1} x_{2}\left(x_{0}^{2} x_{2}^{2}, x_{0} x_{1}^{3}, x_{0} x_{1}^{2} x_{2}, x_{1}^{3} x_{2}\right), x_{0} x_{1} x_{2}\left(x_{0}^{3} x_{2}, x_{0}^{2} x_{1}^{2}, x_{0} x_{1} x_{2}^{2}, x_{1}^{3} x_{2}\right), x_{0} x_{1} x_{2}\left(x_{0}^{3} x_{1}, x_{0}^{2} x_{2}^{2}\right.$, $\left.x_{0} x_{1} x_{2}^{2}, x_{1}^{3} x_{2}\right), x_{0} x_{1} x_{2}\left(x_{0}^{2} x_{1}^{2}, x_{0}^{2} x_{2}^{2}, x_{1}^{4}, x_{1}^{3} x_{2}\right), x_{0} x_{1} x_{2}\left(x_{0}^{4}, x_{0} x_{1}^{3}, x_{0} x_{2}^{3}, x_{1}^{2} x_{2}^{2}\right), x_{0} x_{1} x_{2}\left(x_{0}^{4}, x_{0} x_{2}^{3}, x_{1}^{4}, x_{1} x_{2}^{3}\right)$.

$\underline{d=8}: x_{0} x_{1}\left(x_{0}^{4} x_{2}^{2}, x_{0}^{3} x_{1}^{3}, x_{0} x_{1} x_{2}^{4}, x_{1}^{4} x_{2}^{2}\right), x_{0} x_{2}\left(x_{0}^{3} x_{2}^{3}, x_{0}^{2} x_{1}^{2} x_{2}^{2}, x_{0} x_{1}^{4} x_{2}, x_{1}^{6}\right)$

$\underline{d=9}: x_{0} x_{1} x_{2}\left(x_{0}^{3} x_{2}^{3}, x_{0}^{2} x_{1}^{2} x_{2}^{2}, x_{0} x_{1}^{4} x_{2}, x_{1}^{6}\right), x_{0} x_{1} x_{2}\left(x_{0}^{3} x_{1}^{3}, x_{0}^{3} x_{2}^{3}, x_{0}^{2} x_{1}^{2} x_{2}^{2}, x_{1}^{3} x_{2}^{3}\right), x_{0} x_{1} x_{2}\left(x_{0}^{6}, x_{0}^{2} x_{1}^{2} x_{2}^{2}, x_{1}^{6}, x_{2}^{6}\right)$. 


\section{Acknowledgement}

The first author was partially supported by MTM2013-45075-P. The first author of this paper warmly thanks Emilia Mezzetti for interesting conversations and many ideas developed in this paper.

\section{References}

[1] Brenner, H., Kaid, A. (2007). Syzygy bundles on $\mathbb{P}^{2}$ and the Weak Lefschetz property. Illinois J. Math. 51:1299-1308.

[2] Gelfand, I. M., Kapranov, M. M., Zelevinsky, A. V. (1994). Discriminants, Resultants and Multidimensional Determinants. Boston: Birkhäuser.

[3] Grayson, D. R., Stillman, M. E. Macaulay2, a software system for research in algebraic geometry. Available at: http://www.math.uiuc.edu/Macaulay2/

[4] Mezzetti, E., Miró-Roig, R. M. (2016). The minimal number of generators of a Togliatti system. Annali di Matematica Pura ed Applicata 195:2077-2098. DOI: 10.1007/s10231-016-0554-y.

[5] Mezzetti, E., Miró-Roig, R. M. (2016). Togliatti systems and Galois coverings, Preprint, arXiv:1611.05620.

[6] Mezzetti, E., Miró-Roig, R. M., Ottaviani, G. (2013). Laplace equations and the weak Lefschetz property. Canad. J. Math. 65:634-654.

[7] Michałek, M., Miró-Roig, R. M. (2016). Smooth monomial Togliatti systems of cubics. J. Comb. Theory, Ser. A 143: 66-87. DOI: 10.1016/j.jcta.2016.05.004.

[8] Migliore, J., Miró-Roig, R. M. (2003). Ideals of general forms and the ubiquity of the Weak Lefschetz property. J. Pure Appl. Algebra 182:79-107.

[9] Migliore, J., Miró-Roig, R. M., Nagel, U. (2011). Monomial ideals, almost complete intersections, and the Weak Lefschetz property. Trans. Am. Math. Soc. 363(1):229-257.

[10] Perkinson, D. (2000). Inflections of toric varieties. Michigan Math. J. 48:483-515.

[11] Stanley, R. (1980). Weyl groups, the hard Lefschetz theorem, and the Sperner property. SIAM J. Algebraic Discrete Methods 1:168-184.

[12] Togliatti, E. (1929). Alcuni esempi di superfici algebriche degli iperspazi che rappresentano un'equazione di Laplace. Commun. Math. Helvetici 1:255-272.

[13] Togliatti, E. (1946). Alcune osservazioni sulle superfici razionali che rappresentano equazioni di Laplace. Ann. Mat. Pura Appl. (4) 25:325-339.

[14] Watanabe, J. (1987). The Dilworth Number of Artinian Rings and Finite Posets with Rank Function, Commutative Algebra and Combinatorics, Advanced Studies in Pure Mathematics, Vol. 11. North Holland, Amsterdam: Kinokuniya Co., pp. 303-312. 